\title{
Human studies of mitochondrial biology demonstrate an overall lack of binary sex differences: A multivariate meta-analysis
}

\author{
Alex Junker ${ }^{1}$, Jennifer Wang ${ }^{1}$, Gilles Gouspillou ${ }^{2}$, Johannes K. Ehinger ${ }^{3}$, Eskil Elmér ${ }^{3}$, \\ Fredrik Sjövall ${ }^{3}$, Kelsey Fisher-Wellman ${ }^{4,5}$, P. Darrell Neufer ${ }^{4}$, Anthony J.A. Molina ${ }^{6}$, \\ Luigi Ferrucci ${ }^{7}$, Martin Picard ${ }^{1,8,9}$
}

${ }^{1}$ Division of Behavioral Medicine, Department of Psychiatry, Columbia University Irving Medical Center, New York, NY, USA.

${ }^{2}$ Department of Kinanthropology, Université du Québec à Montreal, Montreal, Canada.

${ }^{3}$ Mitochondrial Medicine, Department of Clinical Sciences, Lund University, Lund, Sweden.

${ }^{4}$ East Carolina Diabetes and Obesity Institute, East Carolina University, Greenville, NC, USA.

${ }^{5}$ Department of Physiology, Brody School of Medicine, East Carolina University, Greenville, NC, USA.

${ }^{6}$ Department of Medicine, University of California San Diego, La Jolla, CA, USA.

${ }^{7}$ Translational Gerontology Branch, National Institute on Aging, National Institutes of Health, Baltimore, MD, USA.

${ }^{8}$ Department of Neurology, H. Houston Merritt Center and Columbia University Translational Neuroscience Initiative, Columbia University Irving Medical Center, NY, USA.

${ }^{9}$ New York State Psychiatric Institute, NY, USA.

Corresponding author: martin.picard@ columbia.edu

630 West 168th Street, Box 172

New York, NY 10032

646-774-5026 


\section{Nonstandard abbreviations}

$\mathrm{CI}$ - complex I, NADH dehydrogenase

CII - complex II, succinate dehydrogenase

CIII - complex III, ubiquinol-cytochrome c reductase

CIV - complex IV, cytochrome c oxidase

$\mathrm{CV}$ - complex V, ATP synthase

CS - citrate synthase

GHT - gender-affirming hormone therapy

mtDNA - mitochondrial DNA

mtDNAcn - mitochondrial DNA copy number

MRS - magnetic resonance spectroscopy

OxPhos - Oxidative phosphorylation

PBMCs - peripheral blood mononuclear cells

ROS - reactive oxygen species

TGD - transgender and gender diverse 


\begin{abstract}
Mitochondria are maternally inherited organelles that play critical tissue-specific roles, including hormone synthesis and energy production, that influence development, health, and aging. However, whether mitochondria from women and men exhibit consistent biological differences remains unclear, representing a major gap in biomedical knowledge. This meta-analysis systematically examined 4 domains and 6 subdomains of mitochondrial biology (total 39 measures), including mitochondrial content, respiratory capacity, reactive oxygen species (ROS) production, morphometry, and mitochondrial DNA copy number. Standardized effect sizes (Hedge's g) of sex differences were computed for each measure using data in 2,258 participants (51.5\% women) from 50 studies. Only two measures demonstrated aggregate binary sex differences: higher mitochondrial content in women $\left(g=0.20, \chi^{2} \mathrm{p}=0.01\right)$, and higher ROS production in skeletal muscle in men $\left(g=0.49, \chi^{2} \mathrm{p}<0.0001\right)$. Sex differences showed weak to no correlation with age or BMI. Studies with small sample sizes tended to overestimate effect sizes $(r=-0.17, p<0.001)$, and sex differences varied by tissue examined. Our findings point to a wide variability of findings in the literature concerning possible binary sex differences in mitochondrial biology. Studies specifically designed to capture sex- and gender-related differences in mitochondrial biology are needed, including detailed considerations of physical activity and sex hormones.
\end{abstract}

\title{
Keywords
}

Mitochondrion; sex differences; sexual dimorphism; respirometry; mtDNAcn 


\section{Introduction}

Mitochondria are at the origin of multicellular life that gave rise to animal sexual reproduction, and to breathing, walking, and thinking homo sapiens (1). Mitochondria have shaped human physiology via energy transformation through oxidative phosphorylation (OxPhos) and via their multiple signaling functions (2). Over the last four decades, mitochondria have been implicated as a cause of rare (3) and common diseases including metabolic disorders, neurodegeneration, and cancer $(4,5)$. Mitochondria may also regulate the aging process in animals and humans (6-10), and serve as biomarkers of stress and disease $(11,12)$.

Consequently, the mitochondrion has become the most studied organelle in the biomedical sciences (13). This scientific focus on mitochondria emphasizes the need to understand why and how much mitochondria differ between individuals (inter-individual variation) among the diverse human population. Remarkably, as a field, we still do not know if, and to what extent, mitochondria display systematic binary sex differences between women and men. Resolving this question is paramount to developing clinically useful metrics of mitochondrial biology and to building comprehensive models of human health that incorporate bioenergetics.

Mitochondria are complex, multi-functional organelles. Within the cell, they have a life cycle (14), interact with one another through fusion processes $(15,16)$, and serve dozens of intracellular functions including ATP synthesis, reactive oxygen species (ROS) production, calcium handling, apoptotic signaling, redox homeostasis, hormone synthesis, and lipid and amino acid metabolism, among others (13). To accomplish specialized tasks in different cell types, mitochondria functionally specialize and acquire distinct tissue-specific proteomes and functional phenotypes $(17,18)$. For these reasons, single static molecular measures, such as mitochondrial DNA (mtDNA) copy number (mtDNAcn), provide little to no information about their functional capacity or complex biological phenotypes (19). Functionally meaningful mitochondrial metrics are best captured by dynamic measurements of functional capacity, such as respiratory capacity and efficiency (20), or ROS emission in the presence of specific metabolic substrates (21). Moreover, because mitochondria are multifunctional, mitochondrial phenotyping is most accurately achieved by probing in parallel multiple metrics of mitochondrial content and specific functions (e.g., (22)). 
Recognizing the multiple roles and functions that mitochondria play in the organism highlights the inadequacy of the popular term "mitochondrial function." By analogy, in human biomedical research, we do not speak of assessing "human function" - a category too imprecise to have empirical value - and instead rely on specific domains and subdomains of biology (locomotion, cognition, digestion). Similarly, our current understanding of mitochondrial biology makes it clear that speaking of "mitochondrial function" lacks the required specificity. Operationalizing different domains of mitochondrial biology to cover the major properties and unique functions of mitochondria provides a framework to systematically explore sex differences with the required degree of specificity (Figure 1).

Three factors highlight the deep-rooted biological links between sex as a biological attribute and mitochondria. First, mammalian mitochondria are maternally inherited - meaning they are passed down solely through the oocyte (23). Second, an underappreciated fact is that the rate limiting step in the synthesis of all sex hormones, including estrogens, progestins, and testosterone (24) takes place in mitochondria, predominantly within the ovaries and testes. Indeed, the first enzymatic steps to the synthesis of all steroid hormones, which also includes glucocorticoids and mineralocorticoids, take place in the mitochondrial matrix (25). Third, mitochondria contain receptors for sex hormones. Both the estrogen receptor $(\operatorname{Er} \beta)(26)$, and the androgen receptor (AR) (27) translocate to the mitochondrial matrix where they interact with mtDNA and influence multiple domains of mitochondrial biology. Thus, mitochondria contain the molecular machinery to i) produce and ii) sense the canonical hormones of sexual differentiation.

Previous studies have partially examined sex differences in mitochondria within different species, but we lack an overarching answer that considers the breadth of mitochondrial biology and of sexual characteristics in humans. In rats and mice, compared to males, mitochondria from females produce more energy and fewer ROS (28). However, it has been argued that the biological and social complexities of human gender/sex (29-31) make animal studies inadequate to explore sex differences in human mitochondrial biology. Human studies measuring sex differences in some aspects of mitochondrial biology have reported higher citrate synthase (CS, a marker of mitochondrial content) activity and greater antioxidant capacity in women's mitochondria $(28,32)$, and comparable maximal respiration rates between binary sexes (33). Increasing evidence also suggests that sex hormones have a strong impact on mitochondrial 
biology independent of chromosomal sex and/or sex assigned at birth (34). Together, these findings highlight the need for a concerted community effort to systematically resolve the nature and magnitude of sex differences in mitochondrial biology among humans.

To address this need, we performed a quantitative, multivariate meta-analysis of available data on binary human sex differences, informed by a framework that incorporates multiple domains of human mitochondrial biology. When possible, we also explored whether methodological approaches (energetic substrates, normalization approach, isolated mitochondria vs. permeabilized cells/fibers methods) and individual traits (physical activity, age, and BMI) moderate the observed sex differences.

\section{Methods}

\section{Outcomes}

Mitochondrial biology was organized into four overarching domains: content, functions, mtDNAcn, and morphometry. Mitochondrial morphometry included two sub-domains, mitochondrial size and mitochondrial volume density. Mitochondrial content was assessed as CS activity and OxPhos complex protein content (CI - CV). Mitochondrial functions included four subdomains including: i) OxPhos enzymatic activity (CI - CV), ii) in vivo mitochondrial OxPhos capacity assessed through 31P-MRS, iii) ROS production, and iv) respiration. Mitochondrial respiratory capacity data was compared according to normalization method (normalized to mitochondrial content, tissue mass, or per cell), type of preparation (permeabilized fibers/cells, or isolated mitochondria), and respiratory state (state 3 phosphorylating respiration, or Leak/State 4 respiration). This organization is depicted in Figure 1b.

\section{Literature search}

This meta-analysis was conducted according to the Preferred Reporting Strategies for Systematic Reviews and Meta-Analyses (PRISMA) guidelines (35). Authors J.W. and A.J. searched Medline via PubMed for eligible humans studies using two approaches: 1) a narrow search for studies that actively considered sex differences in mitochondrial biology, using the following search terms: mitochondria AND ("mitochondri* function" OR "functional capacity" OR respiration OR "ROS product*" OR "reactive oxygen species product*" OR "antioxidant 
activity" OR "ATP synthesis" OR “calcium uptake” OR “calcium handling” OR "electron transport chain" OR fusion OR fission OR "mitochondrial content") AND ("men and women" OR "sex difference" OR "sex differences" OR "sexual dimorphism" OR "sex effects" OR "gender difference" OR "gender differences"); 2) a broad search for studies of mitochondrial biology, regardless of whether there was initial analysis by sex, using the following terms and filters: "mitochondrial function" NOT "review" ("muscle" OR "leukocytes" OR "brain"), using the filters Journal Articles, English, Human, Male, and Female. The last search was completed on July 17th, 2020. Investigators of included studies were also asked to recommend other relevant publications. Finally, the meta-analysis was advertised on social media and the community at large asked to suggest studies for consideration of inclusion.

\section{Inclusion/exclusion criteria}

Criteria were established prior to searches and included only peer-reviewed articles, published in English, that examined at least one domain of mitochondrial biology (as defined above): mitochondrial content, OxPhos complex protein abundance or activity, in vivo magnetic resonance spectroscopy, respiration, ROS production, mtDNAcn, and mitochondrial volume or size. As calculations of standardized mean differences can be unreliable if the comparison groups sample sizes are severely imbalanced (36), studies had to contain no more than three times as many participants of one sex as another. Only studies of women and men without known illnesses (i.e., healthy controls) were included. Review articles and studies in animal models or cell culture were excluded. Studies of post-mortem tissues were included for their unique ability to enable mitochondrial measurements in otherwise inaccessible tissues such as the brain and cardiac muscle.

\section{Data extraction}

The corresponding author(s) of each eligible article were contacted by email to request sex disaggregated data (mean, n, and SD), using a standardized template (Supplemental File 1). Follow-up emails were sent biweekly; after the third nonresponse, the first or senior author was also contacted. Returned data was entered into the data log, organized by domains of mitochondrial biology. Additional information was gathered for each study, including (where applicable) respiratory substrates used, methodological details (e.g., isolated mitochondria or permeabilized cells/fibers), average group age, BMI, and race, as available. The compiled data 
used in this meta-analysis is available as a resource in Supplemental File 2 in the Open Research section of this article.

\section{Characteristics of included studies}

Our searches yielded 1,212 publications in total. After screening abstracts, 275 full-text articles were evaluated for inclusion. Of these, 225 were excluded because they were either animal studies $(n=6)$, reviews $(n=7)$, lacked either women or men participants $(n=39)$, did not directly measure a functional or morphometric domain of mitochondrial biology (e.g., measured TFAM or UCP-1 protein abundance or gene expression) $(n=41)$, lacked healthy controls $(n=$ 17), or had inadequate sample sizes ( $\mathrm{n}=98$, see section "Inclusion/exclusion criteria"). An additional 17 studies were excluded because we failed to establish contact with the author, or sex-disaggregated data was not available. A detailed flowchart of the PRISMA-guided search process is available in Figure 2.

A total of 50 studies were included, resulting in a total of 2,268 participants $(1,163$ women and 1,105 men). Publication dates ranged from 1992 to 2021, with 23 studies (46\%) published within the past 5 years. The average year of publication for included studies was 2013, compared to 2008 for unavailable studies, showing that data was more difficult to obtain from older studies. The average participants age ranged from 4 to 85 years (group mean), with almost half the studies (47\%) with averages between 20-39 years. Average BMI was reported for $84 \%$ of studies and ranged from 22 to 34, with 55\% of participant BMIs categorized as healthy under current guidelines $(\mathrm{BMI}<25)$. $78 \%$ of studies did not report data on race or ethnicity. Of the six studies reporting racial data, three reported the race of each participant, and three reported solely the percentage of white participants. In these studies, the racial composition of participants ranged from 60-93\% white, 0-30\% Asian, 0-10\% Black, and 0-5\% Hispanic. No study reported gender/sex data beyond a binary sex designation (female or male). It is assumed that the vast majority of participants in this dataset are cisgender (i.e., their gender identity aligns with their sex assigned at birth), although this cannot be ascertained from the available data. This design limitation precludes interpretation of the relevance of these results for transgender and gender diverse (TGD) individuals (37). All studies reported gender/sex data as either a female or male designation, limiting analyses of sex only as a binary variable. 
Physical activity directly influences several domains of mitochondrial biology $(38,39)$. Physical activity was an available variable in 5 studies: 4 comparisons of mitochondrial biology pre- vs. post-exercise interventions, and 2 comparisons of individuals with different activity levels (e.g., sedentary vs. active).

\section{Data synthesis and statistical analysis}

PRISMA guidelines were consulted throughout the meta-analysis process to ensure standardized methodology. Effect sizes for each subdomain and individual measures were calculated as the standardized mean difference (Hedge's $g$ ) between binary sexes for each measure of mitochondrial biology. A $g$ value of \pm 1.0 indicates that the two sexes differed by one standard deviation (S.D.). Hedge's g was used as it includes a correction factor that outperforms Cohen's $d$ for small sample sizes (under $\mathrm{n}=20$ ) (36). As no field-specific effect size interpretation guidelines yet exist for mitochondrial biology, guidelines were adapted from a recent meta-analysis on metabolic syndrome components (40), whose cutoffs are comparable to those in other fields $(41,42)$. Effect sizes were considered negligible when falling between [absolute values] 0-0.19, small between 0.20-0.39, medium between 0.40-0.79, large between $0.8-0.99$, and very large $>1.00$.

Forest plots show Hedge's g with 95\% confidence intervals (C.I.) with distinct background colors to indicate large effect sizes $(g>+/-0.8$; yellow for higher in women, blue for higher in men). Results are organized by domain of mitochondrial biology, and secondarily by OxPhos complex, methodological approach, and specific measure, as appropriate. Effect sizes are displayed as calculated for all measures excluding MRS measures of phosphocreatine (PCr) time constant, for which the displayed effect size is the inverse of the calculated value (since time constant is considered roughly inverse to mitochondrial function), displaying the inverse of the measure allows higher values to be interpreted as 'better' function, aligning its interpretation with the rest of the forest plot.

For articles containing multiple reported measures with high biologic similarity (e.g., OxPhos CI+II and ETS CI+II measured in the same participants and tissues using the same methods), only one measure was included, selected based on methodological compatibility with other studies, which contributes to keep relative study weights comparable. As studies varied by more than two orders of magnitude in sample sizes, we chose to calculate an unweighted pooled 
estimate for each metric. Using this approach, each study has an equal weight on the overall estimate, rather than reflecting only the largest studies. Of the 50 original articles included, only $7(14 \%)$ reported sex differences as a primary outcome, thus minimizing the risk of publication bias (i.e., studies showing significant sex differences would be preferentially published, and studies with non-significant ones would remain unpublished) for our primary outcome of sex differences.

For each sub-domain, the proportion of studies/measurements that were either 'higher in women' or 'higher in men' was compared using a $\chi^{2}$ tests of equal proportions where the null hypothesis is that an equal number of studies on either sides of the $g=0$. All statistical tests were performed in Prism (version 8.4.3) or Excel (version 16.52), using two-sided distributions, with the $\alpha$ level set to 0.05 .

\section{Results}

\section{Mitochondrial content is higher in women}

Mitochondrial content was measured in 13 studies for a total of 27 measures (26 CS, one cardiolipin) across 264 participants (126 women) and 13 tissues (Figure 3a). Women had higher mitochondrial content in most studies (20 of $\left.26, \chi^{2} \mathrm{p}=0.01\right)$ for an overall estimate slightly higher in women (average $\mathrm{g}=0.20$ ).

Tissue specificity. Mitochondrial content demonstrated potential tissue-specificity (Figure 3b). All isolated leukocyte cell types showed higher CS activity in women (from $\mathrm{g}=$ 0.20 to 1.52 ). Women also had higher CS activity in white adipose tissue (WAT) and brain tissue, ( $g=0.49$ and 0.88 , respectively). However, each of these tissues (isolated leukocytes, WAT, and brain tissue) was measured in one study. Tissues measured across multiple studies, such as combined peripheral blood mononuclear cells (PBMCs) and skeletal muscle, demonstrated mixed sex effects by study and no overall sex differences ( $g=-0.05$ and -0.20 , respectively). Platelets were measured in one study and showed almost equal content in women and men $(g=0.01)$. 
Exercise. One study assessing exercise in elderly individuals found that men had higher CS activity than women pre-exercise ( $g=-1.25$, very large effect size), a difference marginally accentuated post-exercise ( $\mathrm{g}=-1.37$, very large) (Figure 3c).

\section{OxPhos protein abundance does not exhibit consistent sex difference}

OxPhos complex protein abundance was measured in six studies for a total of 32 measures (CI, 8; CII, 5; CIII, 8; CIV, 3; CV, 8) across 87 people (42 women) and two tissues (Figure 3d). The measures were evenly distributed between higher in women or men for a negligible overall effect (average $g=-0.19, \chi^{2} \mathrm{p}=0.30$ ). Most studies found higher $\mathrm{CI}$ abundance in women, but studies reporting higher CI abundance in men had larger effect sizes, resulting in a negligible CI-specific estimate (average $g=-0.14$ ). Similarly, measures of CII abundance were split between higher in women or men, for an overall estimate slightly higher in men $(g=-0.28)$. A stronger effect was observed in both CIII and CV abundance, which were higher in men ( $g=-0.42$ and $g=-0.39$, respectively). In contrast, CIV abundance was approximately equal in women and men $(\mathrm{g}=0.07)$, indicating potential OxPhos complexspecific sex differences.

Tissue specificity. Skeletal muscle OxPhos abundance showed wide variation in both strength and direction of differences, for every OxPhos complex, resulting in a skeletal musclespecific effect size of -0.27 (small, higher in men) (Figure 3e). In contrast, PBMC protein abundance was higher in men for all OxPhos complexes (from $\mathrm{g}=-0.61$ to $\mathrm{g}=-1.18$ ), resulting in a large leukocyte-specific effect size of -0.81 (higher in men). However, all PBMC measures came from one study, making it impossible to disentangle tissue-specific from method-specific factors.

Exercise. One study found higher protein abundance in active women than active men across all complexes (from $g=0.27$ to $g=1.00$ ) (Figure 3f). Sedentary men had higher protein abundance than sedentary women at baseline (from $g=-0.03$ to $g=-0.48$ ), but post-exercise intervention measures showed protein abundance was comparable between sexes (from $\mathrm{g}=-0.03$ to $\mathrm{g}=0.25)$.

\section{OxPhos enzyme activities do not exhibit consistent sex differences}


Respiratory chain complex enzymatic activity was measured in 6 studies for a total of 40 measures (CI, 11; CII, 15; CIII, 1; CIV, 12; CV, 1) across 145 people (63 women) and 11 tissues (Figure 4a). The measures were evenly distributed between those higher in women or men and showed no overall effect $\left(g=-0.01 ; \chi^{2} \mathrm{p}=0.31\right)$. In analyses disaggregated by OxPhos complexes, CI activity measures showed no overall effect. Measures higher in women tended to demonstrate stronger effect sizes (up to $g=1.36$ ), but the even distribution of values resulted in OxPhos complex-specific average effect sizes only negligibly higher in either women or men.

Tissue specificity. CII activity in isolated leukocyte subtypes was consistently higher activity in women (Figure 4a). Cell subtype data available from one study included both naïve and memory states of CD4+ and CD8+ T cells, two states well known to have different mitochondrial profiles (43). Tissue-specific average effect sizes concur, with six of the eight leukocyte subtypes showing higher activity in women (Figure 4b). In contrast, mixed PBMCs (a mixture of lymphocytes and monocytes) were only negligibly higher in men $(g=-0.16)$. Skeletal muscle OxPhos enzyme activity was moderately higher in men $(g=-0.58)$, while brain tissue was slightly higher in women $(g=0.39)$.

Exercise. One study of physical activity found that an exercise intervention tended to accentuate the higher skeletal muscle CII enzymatic activity ( $g=-1.16$ and -1.42 , respectively) (Figure 4c).

Sex differences of in vivo mitochondrial OxPhos capacity may depend on the MRS metric used

In vivo phosphorus magnetic resonance spectroscopy $\left({ }^{31} \mathrm{P}-\mathrm{MRS}\right)$ was used to assess mitochondrial oxidative phosphorylation capacity in 14 studies for a total of 27 measures (PCr resynthesis rate, 4; $\mathrm{PCr}$ rate constant, 16; $\mathrm{PCr}$ time constant, 6) in skeletal muscle across 1,163 people (611 women) (Figure 4c). There was no data available for tissues other than skeletal muscle, or exercise-related analyses. Across all metrics, there is no evidence of sex differences (average $\mathrm{g}=0.00, \chi^{2} \mathrm{p}=0.78$ ). Considered independently, both $\mathrm{PCr}$ rate constant and time constant showed negligible sex differences (average $\mathrm{g}=-0.13$ and 0.10 , respectively). In contrast, all measures of $\mathrm{PCr}$ resynthesis rate were higher in women ranging from medium to large effect sizes ( $g=0.57$ and 0.87 , respectively), for a measure-specific average effect size of $g$ $=0.67$ (medium). Studies of PCr rate and time constant had significantly larger sample sizes (up 
to 685 people per measure) compared to studies of $\mathrm{PCr}$ resynthesis rate ( $\mathrm{n}=9$ to 30 participants), which may contribute to the discrepancy in reported effect sizes.

\section{Mitochondrial ROS production is higher in men's skeletal muscle}

Mitochondrial ROS production was measured in 6 studies for a total of 31 measures across 152 people (90 women) (Figure 5a). There was an overall medium effect size showing greater ROS production in men than women $\left(g=-0.49, \chi^{2} \mathrm{p}<0.0001\right)$. The vast majority of measures were higher in men $(88 \%)$, with effect sizes ranging from negligible $(g=-0.03)$ to very large $(g=-1.61)$. This effect was consistent across several experimental conditions, including in the presence of oligomycin $(g=-0.47)$, rotenone $(g=-0.07)$, succinate $(g=-0.49)$, or other assorted carbohydrate or lipid substrates $(g=-0.56)$.

Tissue specificity. Only one study measured ROS in combined PBMCs, finding negligibly higher production in men $(g=-0.16)$ (Figure 5b). The remaining measures (30 of 31) were performed in skeletal muscle and yielded an average medium effect size $(\mathrm{g}=-0.50)$ indicating higher ROS production in men.

\section{Sex differences in phosphorylating respiration are sensitive to tissue and methodology}

A total of 22 studies measured phosphorylating (state 3 ) respiration, utilizing a range of methodological and analytic approaches. Phosphorylating respiration was normalized to CS in 6 studies, resulting in 27 measures across 120 people (63 women) and three tissues (Figure 6a). The majority of these (27 of $37,72 \%$ ) were measured in permeabilized cells and myofibers and showed negligible sex differences in CI-stimulated (0.10), CII-stimulated (-0.07), and CI+II (0.03 ) respiration, for no sex difference overall (average $g=0.06$ ). The remaining 10 measures were evaluated in mitochondria isolated from skeletal muscle. Women had higher respiration for $\mathrm{CI}+\mathrm{II}$ and CI-stimulated state 3 respiration $(\mathrm{g}=0.26$ and 0.56 , respectively). In contrast, men had higher CII-driven respiration $(\mathrm{g}=-0.68)$, indicating that sex differences may be specific to OxPhos complexes. Overall, measures of respiration normalized to CS show no sex difference whether collected in permeabilized fibers or isolated mitochondria $(g=0.16)$, for overall no sex difference $\left(g=0.10, \chi^{2} \mathrm{p}=0.31\right)$.

An additional 5 studies normalized phosphorylating respiration to cell count, resulting in 29 measures (CI, 8; CII, 6; CI+II, 11; CIV , 4; 26) from 87 participants (46 women) and three 
tissues (Figure 6b). Respiration was predominantly higher in men across all states, with average effect sizes ranging from negligible $(\mathrm{g}=-0.13, \mathrm{CI}+\mathrm{II})$ to moderate $(\mathrm{g}=-0.51, \mathrm{CI})$, for an overall estimate slightly, but significantly, higher in men $\left(g=-0.30, \chi^{2} \mathrm{p}=0.01\right)$.

Phosphorylating respiration was normalized to tissue mass in 12 studies, resulting in 45 measures across 302 participants (161 women) and two tissues (Figure 6c). Most of these measures (36 of 45, 80\%) were collected from respirometry performed in permeabilized myofibers or WAT cells and demonstrated no sex differences in CI-, CII-, or CI+II-stimulated respiration $(g=-0.08,-0.11$, and 0.08 , respectively), for an overall absence of sex differences (average $\mathrm{g}=-0.02$ ). The remaining 9 measures were from 3 studies that performed respirometry in mitochondria isolated from skeletal muscle. Average respiration was higher in men across all states, with effect sizes ranging from small to very large (CI, g = -0.28; CII, -1.03; CI+II, -0.34). Overall, phosphorylating respiration normalized to mass was moderately higher in men when measured in isolated mitochondria $(\mathrm{g}=-0.44)$, but not in permeabilized cells/fibers, for an overall lack of sex differences $\left(g=-0.12, \chi^{2} \mathrm{p}=0.56\right)$.

Tissue specificity. These measures of phosphorylating respiration demonstrated potential tissue-specific sex differences (Figure 6d). Regardless of methodological approach, women had higher phosphorylating respiration in WAT $(\mathrm{g}=0.66$, permeabilized cells; $\mathrm{g}=0.85$, isolated mitochondria). Platelet respiration was higher in men across all normalizations ( $\mathrm{g}=-0.61$, to CS; $\mathrm{g}=-0.41$, to cell). However, sex differences in other tissues differed by method: women had higher respiration in PBMCs when normalized to CS (average $g=0.73$ ), but men had higher respiration when normalized to cell count (average $g=-0.49$ ). Skeletal muscle respiration normalized to CS tended to be negligibly higher in women when measured in isolated mitochondria (average $\mathrm{g}=0.16$ ), but measures in permeabilized myofibers were higher in men (average $\mathrm{g}=-0.29$, small effect size). In contrast, all measures of skeletal muscle respiration normalized to mass were higher in men (ranging from $\mathrm{g}=-0.06$ to $\mathrm{g}=-0.44$ ). When measures from both permeabilized fibers and isolated mitochondria are considered together, overall skeletal muscle respiration does not differ between binary sexes (from $g=-0.01$ to $g=-0.16$ ).

\section{Sex differences in mtDNAcn are tissue-specific}

The number of mtDNA copies per cell, or mtDNAcn, was measured in 10 studies for a total of 42 measures across 308 people (146 women) and a wide range of tissues (Figure 7a). 
There were no consistent binary sex differences in mtDNAcn (average $g=0.01, \chi^{2} \mathrm{p}=0.75$ ) and, in contrast to other domains, there were 0 measures with strong sex effects for either women or men $(\mathrm{g}>/=0.8)$.

Tissue specificity. Women had higher mtDNAcn across WAT and whole blood $(\mathrm{g}=0.40$ and 0.30, respectively) (Figure 7b). Several immune cell subtypes also showed higher mtDNAcn in women, including B cells $(\mathrm{g}=0.50)$ and CD8+ \& CD4+CMEM cells $(\mathrm{g}=0.58$ and 0.36 , respectively). In contrast, men had higher mtDNAcn for both neutrophils $(\mathrm{g}=-0.73)$ and monocytes (-0.37). However, all measures of mtDNAcn in immune cell subtypes came from one study, precluding interpretation on the effects of tissue-specific or method-specific factors.

\section{Skeletal muscle mitochondrial morphometry does not exhibit sex differences}

Skeletal muscle mitochondrial volume density was measured in 7 studies for a total of 13 measures across 224 people (121 women) (Figure 8a). Only one measure showed substantially higher volume density in women $(g=1.15)$, while four measures demonstrated substantially higher volume density in men (ranging from $g=-0.96$ to -4.98 ). Overall, mitochondrial volume density appeared to be higher in men's skeletal muscle $\left(g=-0.60, \chi^{2} p=0.28\right)$. However, this difference may be exaggerated by the presence of an outlier with an effect size almost three times larger than any other measure of volume density $(g=-4.98)$. When this measure is removed, the average overall mitochondrial volume density decreases by almost half, but remains higher in men $(\mathrm{g}=-0.31)$.

Skeletal muscle mitochondrial size was measured using electron microscopy in 5 studies for a total of 8 measures across 89 people (49 women) (Figure 8c). The measures were evenly distributed between being higher in women or men $\left(\chi^{2} \mathrm{p}=0.52\right)$. The overall estimate was higher in men $(g=-0.44)$, likely due to one measure with a very large effect size $(g=-3.52)$. When this measure is removed, there is no sex difference in mitochondrial size (average $\mathrm{g}=-0.10$ ).

Exercise. Two studies found higher volume density in men when assessed post-exercise intervention ( $g=-0.21$ to -0.99 ) (Figure 8b), but pre-exercise measures varied, with one showing higher density in women $(\mathrm{g}=0.27)$ and one showing higher density in men $(\mathrm{g}=-0.25)$. A third study found higher volume density in men among sedentary individuals $(\mathrm{g}=-1.78)$, but comparable density between sexes in active individuals $(\mathrm{g}=-0.01)$. One study assessed 
mitochondrial size in older individuals and found that women had larger mitochondria pretraining $(\mathrm{g}=0.48)$, but men had larger size post-training $(\mathrm{g}=-0.54)$ (Figure 8d).

Binary sex differences in mitochondrial biology show weak to no association with age and BMI

We systematically evaluated a potential moderating role of age and BMI on all domains of mitochondrial biology described above. Only two domains showed a statistically significant correlation with age: as a study group's average age increased, i) ROS production became higher in men compared to women, and ii) respiration per cell changed from higher in men to showing no sex difference (Figure 9a). However, this dataset is limited in its ability to address this question. Most mitochondrial domains exhibited poor age resolution across the lifespan: for example, most studies of ROS production were conducted in individuals aged 20-30 or 70-80, making our analysis functionally more of a two-group comparison than a true correlation. This poor resolution, along with analyzing multiple tissues and methods together, makes it difficult to interpret whether sex differences in mitochondrial biology are moderated by age.

There were no significant correlations between mitochondrial biology and average group BMI, a limited and imperfect surrogate for body composition or obesity (44) (Figure 9b). The strongest negative correlation was for respiration to cell $(r=-0.59, p=-0.096$, suggesting that as BMI increases, the moderately higher respiration per cell in younger women approaches the null hypothesis. However, the relatively few measures on this analysis (9) provides poor resolution and little confidence in this conclusion. Near-continuous coverage in average BMI was only present for analysis of in vivo MRS and the combined respiration plot, boof which showed almost no correlation ( $\mathrm{r}=0.05$ and -0.03 , respectively).

\section{Influence of sample sizes on sex differences}

To systematically assess the extent to which sample sizes may have influenced effect size estimates of sex differences across domains of mitochondrial biology, we plotted effect sizes as a function of sample size (Figure 10a). For each available measure, as expected (45), studies with smaller sample sizes yielded larger effect size estimates compared with larger studies particularly for three subdomains: MRS $(r=-0.36, p$-value $=0.04)$, mitochondrial respiration $(r=-0.26, p$ value $=0.006)$, and mtDNAcn $(r=-0.38, p$-value $=0.01)$. A similar association between sample 
size and effect size $(r=-0.17$, p-value $<0.001)$ was observed when all measures of mitochondrial biology analyzed together (Figure 10b).

\section{Discussion}

In this meta-analysis of 39 distinct measures of mitochondrial biology from 50 studies combining >2,000 individuals, only two measures demonstrated consistent binary sex differences: higher mitochondrial content in women's WAT and isolated leukocyte subpopulations, and higher ROS production in men's skeletal muscle. Other measures showed inconsistent sex differences with large divergences in strength and direction across studies, experimental conditions (e.g., substrates), and sampled tissues. Sex differences across domains of mitochondrial biology showed weak to no interactions with BMI or age, although the available data offered sparse coverage of the human lifespan limiting the interpretation of these findings. Overall, these results indicate a lack of binary sex differences across most domains of mitochondrial biology, highlighting the need for adequately powered studies specifically designed to define the nature and magnitude of gender/sex and inter-individual differences in mitochondrial biology, and their relation to human health.

Three limitations of the current literature emphasize outstanding gaps in knowledge that poses challenges for the field. First, sex differences in mitochondrial health are heavily dependent on the tissue examined. For instance, women had higher respiration in WAT, while respiration appeared higher for men in platelets. This aligns with ongoing work characterizing biological sex variation within specific organ systems, such as the brain (46-48). Most available data included in this meta-analysis was derived from easily accessible tissues including blood, skeletal muscle, or adipose tissue; thus, whether less-studied tissues exhibit sex differences remains largely unknown. Sex differences may further vary between short-lived (certain blood leukocytes) and long-lived cells (neurons) due to their unequal cumulative exposure to sex hormones or other gendered influences. Second, little remains known about the interaction of sex and age on mitochondria. While the average ages among included studies ranged from 4 to 95 years, $\sim 50 \%$ of included studies mostly covered the third and fourth decades of life. Human aging involves well-documented changes in both sex hormone levels (49) and in mtDNA quality (50-52) but the relative paucity of data on older individuals in this analysis precludes definitive 
conclusions about whether sex differences are age-dependent. Additional work, particularly longitudinal studies, are therefore needed to characterize mitochondrial sex differences across the lifespan. Third, our results suggest that sex differences in mitochondrial biology may be sensitive to methodological (isolated mitochondria vs. permeabilized cells/fibers) and analytic (normalization to CS, cell, or mass) approaches. This sensitivity aligns with previous research showing that the effect of aging on mitochondrial respiratory capacity, ROS emission, and calcium retention capacity was exaggerated in isolated mitochondria compared to permeabilized myofibers (53), suggesting that inherent differences can be revealed or masked by different experimental preparations.

In designing future studies, the dynamic nature of mitochondrial biology should also be considered. For several psychophysiological phenotypes, within-person variation can be equal and up to four times larger than between-person differences shown at the group level (54). Thus, the biological significance of group differences, such as those meta-analyzed here, should be considered in light of how much natural variation can occur over time within individuals. Most available studies measured mitochondrial biology at a single time point; repeated-measures were only available in a small number of intervention studies (usually exercise) such that observed changes were attributed to the intervention's effects. One proof-of-concept n-of-1 study documented 20 - 30\% week-to-week variation in OxPhos enzymatic activities in sorted circulating leukocytes, suggesting substantial within-person variation in mitochondrial biology (22). We also previously found that up to $15 \%$ of the variance in PBMC mitochondrial OxPhos enzymatic activity normalized to mitochondrial content was predicted by psychological states the night before the blood draw (12), suggesting that mitochondria may dynamically adapt over short time frames and in response to more diverse factors than previously conceived. Overall, the natural variation in mitochondrial biology over time largely remains to be defined. Future studies specifically examining sex-related group differences in the context of intra-individual variation are needed to better understand the interplay of mitochondrial biology with different aspects of sex and gender.

Human sex is a complex system of biological features including, but not limited to, hormones, chromosomes, and tissues. Historically, these attributes have been reduced to a binary categorization of sex assigned at birth. Reducing this complexity into the binary "female or male" is a convenient categorization, but growing knowledge indicates that this binary 
categorization occludes our understanding of the true complexity of human sex and gender (often defined as socially-constructed roles and norms) (55-57). For example, one study following transmasculine people on gender-affirming hormone therapy (GHT) found that they had higher ROS production after three months taking testosterone (34). This finding suggests that variation in hormone levels - including between individuals with the same chromosomal sex - could influence mitochondrial behavior regardless of sex assigned at birth. Moreover, well-documented influences of gender as a social position on one's diet (58-60), physical activity (61-64), sleep (65-67), and other health behaviors (68-70) are not captured by a binary sex classification, and may be responsible for physiological differences otherwise superficially attributed to biological sex (30). Sexual characteristics can also vary within a single person over time: gender rolerelated health behaviors change with marital status (71-73), and sex hormones are altered with parental role transitions (74), common conditions like polycystic ovary syndrome (PCOS) (75), and due to menopause or andropause $(49,76)$. On the one hand, it is possible that these additional factors simply add noise to the binary analyses reported above, thus reducing effect sizes. On the other hand, the influence of these factors on mitochondrial health could supersede that of sex assigned at birth, making the binary classification of limited use, possibly obscuring meaningful gender/sex-related variation. Research findings guided solely by a binary sex construct also are of limited applicability to TGD individuals, who represent a non-negligible and increasing fraction of the general population (37), and to intersex individuals $(77,78)$. A complete discussion of the limitations of binary sex constructs are beyond the scope of this metaanalysis but are explored elsewhere $(29-31,79,80)$.

In summary, characterizing human sex differences in mitochondrial biology remains an important frontier and a collective challenge for biomedical scientists. While our meta-analysis reveals small-to-moderate binary sex differences among specific domains of mitochondrial biology, available evidence highlights the need for future adequately-powered, repeatedmeasures studies informed by a more nuanced and accurate understanding of gender/sex influences on mitochondria and cellular bioenergetics.

\section{Author contributions}

J.W., A.J., and M.P. designed research. J.W. and A.J performed the literature review, collected, and organized data. A.J., G.G., J.E., and M.P. analyzed and interpreted data. A.J. prepared the 
figures. A.J. and M.P. drafted the manuscript. All authors edited and commented on the final version of the manuscript.

\section{Acknowledgements}

This meta-analysis was a group effort from investigators across multiple fields including metabolism/diabetes, exercise physiology, skeletal muscle biology, and gerontology. We are grateful to investigators and their team who provided sex-disaggregated data for this crossdisciplinary effort. Work of the authors was supported by FRQS grant 297877 to G.G., the Wharton Fund to A.J. and M.P., and NIH grants MH119336, AG066828, MH122706, and MH123927 to M.P.

\section{Conflict of interest}

None of the authors have a conflict of interest that could be perceived to bias their work and all funding sources have been disclosed. 


\section{References}

1. Lane, N. (2015) The Vital Question: Energy, Evolution, and the Origins of Complex Life, Profile Books

2. Mottis, A., Herzig, S., and Auwerx, J. (2019) Mitocellular communication: Shaping health and disease. Science 366, 827-832

3. Gorman, G. S., Chinnery, P. F., DiMauro, S., Hirano, M., Koga, Y., McFarland, R., Suomalainen, A., Thorburn, D. R., Zeviani, M., and Turnbull, D. M. (2016) Mitochondrial diseases. Nat Rev Dis Primers 2, 16080

4. Yonova-Doing, E., Calabrese, C., Gomez-Duran, A., Schon, K., Wei, W., Karthikeyan, S., Chinnery, P. F., and Howson, J. M. M. (2021) An atlas of mitochondrial DNA genotype-phenotype associations in the UK Biobank. Nat Genet 53, 982-993

5. Wallace, D. C. (2012) Mitochondria and cancer. Nat Rev Cancer 12, 685-698

6. Jang, J. Y., Blum, A., Liu, J., and Finkel, T. (2018) The role of mitochondria in aging. J Clin Invest 128, 3662-3670

7. Latorre-Pellicer, A., Moreno-Loshuertos, R., Lechuga-Vieco, AV., et al. . (2016) Mitochondrial and nuclear DNA matching shapes metabolism and healthy ageing. Nature

8. Trifunovic, A., Wredenberg, A., Falkenberg, M., Spelbrink, J. N., Rovio, A. T., Bruder, C. E., Bohlooly, Y. M., Gidlof, S., Oldfors, A., Wibom, R., Tornell, J., Jacobs, H. T., and Larsson, N. G. (2004) Premature ageing in mice expressing defective mitochondrial DNA polymerase. Nature 429, 417-423

9. Kujoth, G., Hiona, A., Pugh, TD., et al. (2005) Mitochondrial DNA mutations, oxidative stress, and apoptosis in mammalian ageing. Science

10. Ferrucci, L., Gonzalez-Freire, M., Fabbri, E., Simonsick, E., Tanaka, T., Moore, Z., Salimi, S., Sierra, F., and de Cabo, R. (2020) Measuring biological aging in humans: A quest. Aging Cell 19, e13080

11. Hill, B. G., Shiva, S., Ballinger, S., Zhang, J., and Darley-Usmar, V. M. (2019) Bioenergetics and translational metabolism: implications for genetics, physiology and precision medicine. Biol Chem 401, 3-29

12. Picard, M., Prather, A. A., Puterman, E., Cuillerier, A., Coccia, M., Aschbacher, K., Burelle, Y., and Epel, E. S. (2018) A Mitochondrial Health Index Sensitive to Mood and Caregiving Stress. Biol Psychiatry 84, 9-17

13. Picard, M., Wallace, D. C., and Burelle, Y. (2016) The rise of mitochondria in medicine. Mitochondrion 30, 105-116

14. Twig, G., Elorza, A., Molina, A. J., Mohamed, H., Wikstrom, J. D., Walzer, G., Stiles, L., Haigh, S. E., Katz, S., Las, G., Alroy, J., Wu, M., Py, B. F., Yuan, J., Deeney, J. T., 
Corkey, B. E., and Shirihai, O. S. (2008) Fission and selective fusion govern mitochondrial segregation and elimination by autophagy. EMBO J 27, 433-446

15. Labbé, K., Murley, M., \& Nunnari, J. (2014) Determinants and Functions of Mitocondrial Health. . Annual Review of Cell and Developmental Biology.

16. Porat-Shliom, N., Harding, O. J., Malec, L., Narayan, K., and Weigert, R. (2019) Mitochondrial Populations Exhibit Differential Dynamic Responses to Increased Energy Demand during Exocytosis In Vivo. iScience 11, 440-449

17. Rath, S., Sharma, R., Gupta, R., et al. (2021) MitoCarta 3.0: an updated mitochondrial proteome now with suborganelle localization and pathway annotations. .

18. Picard, M., Hepple, R. T., and Burelle, Y. (2012) Mitochondrial functional specialization in glycolytic and oxidative muscle fibers: tailoring the organelle for optimal function. $\mathrm{Am}$ J Physiol Cell Physiol 302, C629-641

19. Picard, M. (2021) Blood mitochondrial DNA copy number: What are we counting?

20. McLaughlin, K. L., Hagen, J. T., Coalson, H. S., Nelson, M. A. M., Kew, K. A., Wooten, A. R., and Fisher-Wellman, K. H. (2020) Novel approach to quantify mitochondrial content and intrinsic bioenergetic efficiency across organs. Sci Rep 10, 17599

21. Anderson, E. J., \& Neufer, P. D. (2006) Type II skeletal myofibers possess unique properties that potentiate mitochondrial $\mathrm{H}(2) \mathrm{O}(2)$ generation.

22. Rausser, S., Trumpff, C., McGill, M. A., Junker, A., Wang, W., Ho, S. H., Mitchell, A., Karan, K. R., Monk, C. E., Segerstrom, S. C., Reed, R. G., \& Picard, M. (2021) Mitochondrial phenotypes in purified human immune cell subtypes and cell mixtures. eLife

23. Giles, R. E., Blanc, H., Cann, H. M., and Wallace, D. C. (1980) Maternal inheritance of human mitochondrial DNA. Proc Natl Acad Sci U S A 77, 6715-6719

24. Selvaraj, V., Stocco, D. M., and Clark, B. J. (2018) Current knowledge on the acute regulation of steroidogenesis. Biol Reprod 99, 13-26

25. Picard, M., McEwen, B. S., Epel, E. S., and Sandi, C. (2018) An energetic view of stress: Focus on mitochondria. Front Neuroendocrinol 49, 72-85

26. Ventura-Clapier, R., Piquereau, J., Veksler, V., and Garnier, A. (2019) Estrogens, Estrogen Receptors Effects on Cardiac and Skeletal Muscle Mitochondria. Front Endocrinol (Lausanne) 10, 557

27. Bajpai, P., Koc, E., Sonpavde, G., Singh, R., and Singh, K. K. (2019) Mitochondrial localization, import, and mitochondrial function of the androgen receptor. J Biol Chem 294, 6621-6634 
28. Ventura-Clapier, R., Moulin, M., Piquereau, J., Lemaire, C., Mericskay, M., Veksler, V., and Garnier, A. (2017) Mitochondria: a central target for sex differences in pathologies. Clin Sci (Lond) 131, 803-822

29. Fausto-Sterling, A. (2012) Sex/Gender: Biology in a Social World, Routledge, New York

30. Richardson, S. (2021) Sex contextualism. Philosophy, Theory, and Practice in Biology

31. Ritz, S. A., Antle, D.M., Côté, J., Deroy, K., Fraleigh, N., Messing, K., Parent, L., StPierre, J., Vaillancourt, C. and Mergler, D. . (2014) First steps for integrating sex and gender considerations into basic experimental biomedical research.

32. Silaidos, C., Pilatus, U., Grewal, R., Matura, S., Lienerth, B., Pantel, J., and Eckert, G. P. (2018) Sex-associated differences in mitochondrial function in human peripheral blood mononuclear cells (PBMCs) and brain. Biol Sex Differ 9, 34

33. Miotto, P. M., McGlory, C., Holloway, T. M., Phillips, S. M., and Holloway, G. P. (2018) Sex differences in mitochondrial respiratory function in human skeletal muscle. Am J Physiol Regul Integr Comp Physiol 314, R909-R915

34. Victor, V. M., Rocha, M., Banuls, C., Rovira-Llopis, S., Gomez, M., and HernandezMijares, A. (2014) Mitochondrial impairment and oxidative stress in leukocytes after testosterone administration to female-to-male transsexuals. J Sex Med 11, 454-461

35. Moher D, L. A., Tetzlaff J, Altman DG. (2009) Preferred Reporting Items for Systematic Reviews and Meta-Analyses: The PRISMA Statement.

36. Cousineau, J.-C. G.-P. a. D. (2018) A review of effect sizes and their confidence intervals, Part I: The Cohen's d family

37. Qi Zhang, M. G., Noah Adams, et al. (2020) Epidemiological considerations in transgender health: A systematic review with focus on higher quality data. International Journal of Transgender Health

38. Memme, J., Erlich, AT., Phukan, G., \& Hood, DA. (2019) Exercise and Mitochondrial Health. The Journal of Physiology

39. Neufer, P. D., Bamman, Marcas M., Muoio, Deborah M., Bouchard, C., Cooper, Dan M., Goodpaster, Bret H., Booth, Frank W., Kohrt, Wendy M., Gerszten, Robert E., Mattson, Mark P., Hepple, Russell T., Kraus, William E., Reid, Michael B., Bodine, Sue C., Jakicic, John M., Fleg, Jerome L., Williams, John P., Joseph, L., Evans, M., Maruvada, P., Rodgers, M., Roary, M., Boyce, Amanda T., Drugan, Jonelle K., Koenig, James I., Ingraham, Richard H., Krotoski, D., Garcia-Cazarin, M., McGowan, Joan A., and Laughlin, Maren R. (2015) Understanding the Cellular and Molecular Mechanisms of Physical Activity-Induced Health Benefits. Cell Metabolism 22, 4-11

40. Mo'ez Al-Islam’ E. Faris, H. A. J., Joud Alsibai and Asma A. Obaideen. (2020) Impact of Ramadan diurnal intermittent fasting on the metabolic syndrome

components in healthy, non-athletic Muslim people aged over 15 years: 
a systematic review and meta-analysis.

41. Brydges, C. R. (2019) Effect Size Guidelines, Sample Size Calculations, and Statistical Power in Gerontology. Innov Aging 3, igz036

42. Gaeta, L., and Brydges, C. R. (2020) An Examination of Effect Sizes and Statistical Power in Speech, Language, and Hearing Research. J Speech Lang Hear Res 63, 15721580

43. Bektas, A., Schurman, S. H., Gonzalez-Freire, M., Dunn, C. A., Singh, A. K., Macian, F., Cuervo, A. M., Sen, R., and Ferrucci, L. (2019) Age-associated changes in human CD4(+) T cells point to mitochondrial dysfunction consequent to impaired autophagy. Aging (Albany NY) 11, 9234-9263

44. Okorodudu, D. O., Jumean, M. F., Montori, V. M., Romero-Corral, A., Somers, V. K., Erwin, P. J., and Lopez-Jimenez, F. (2010) Diagnostic performance of body mass index to identify obesity as defined by body adiposity: a systematic review and meta-analysis. Int J Obes (Lond) 34, 791-799

45. Anderson, S. F., Kelley, K., and Maxwell, S. E. (2017) Sample-Size Planning for More Accurate Statistical Power: A Method Adjusting Sample Effect Sizes for Publication Bias and Uncertainty. Psychological Science 28, 1547-1562

46. McCarthy, M., Arnold, A. . (2011) Reframing sexual differentiation of the brain. Nat Neurosci

47. de Vries, G. J., \& Forger, N.G. (2015) Sex differences in the brain: a whole body perspective. Biology of sex differences

48. Pearse, R. V., 2nd, \& Young-Pearse, T.L. (2019) Lost in translational biology: Understanding sex differences to inform studies of diseases of the nervous system. Brain research

49. van den Beld, K., Zillikens, Lamberts, Egan, van der Lely. (2018) The physiology of endocrine systems with ageing. The Lancet

50. Tian Q, M. A., Oppong R et al. (2021) Mitochondrial DNA copy number and heteroplasmy load correlate with skeletal muscle oxidative capacity by P31 MR spectroscopy. Aging Cell

51. Zhang, R., Wang, Y., Ye, K. et al. . (2017) Independent impacts of aging on mitochondrial DNA quantity and quality in humans. BMC Genomics

52. Ye, K., Lu, J., Ma, F., Keinan, A., \& Gu, Z. (2014) Extensive pathogenicity of mitochondrial heteroplasmy in healthy human individuals. . PNAS

53. Picard, M. e. a. (2010) Mitochondrial functional impairment with aging is exaggerated in isolated mitochondria compared to permeabilized myofibers. Aging Cell 
54. Fisher, A. J., Medaglia, J. D., \& Jeronimus, B. F. (2018) Lack of group-to-individual generalizability is a threat to human subjects research. PNAS

55. Richardson, S. (2021) Sex contextualism. Philosophy, Theory, and Practice in Biology

56. Joel, D. (2021) Beyond the binary: Rethinking sex and the brain. Neuroscience and Biobehavioral Reviews 122, 165-175

57. Joel, D., and Fausto-Sterling, A. (2016) Beyond sex differences: new approaches for thinking about variation in brain structure and function. Philos Trans R Soc Lond B Biol Sci 371, 20150451

58. Rothgerber, H. (2013) Real men don't eat (vegetable) quiche: Masculinity and the justification of meat consumption. Psychology of Men \& Masculinity

59. VanKim, N. A., Corliss, H. L., Jun, H. J., Calzo, J. P., AlAwadhi, M., and Austin, S. B. (2019) Gender Expression and Sexual Orientation Differences in Diet Quality and Eating Habits from Adolescence to Young Adulthood. J Acad Nutr Diet 119, 2028-2040

60. Fidolini. (2021) Eating like a man. Food, masculinities and self-care behavior. Food, Culture, \& Society

61. Bauman, A., Bull, F., Chey, T., Craig, C. L., Ainsworth, B. E., Sallis, J. F., Bowles, H. R., Hagstromer, M., Sjostrom, M., Pratt, M., and Group, I. P. S. (2009) The International Prevalence Study on Physical Activity: results from 20 countries. Int J Behav Nutr Phys Act 6, 21

62. Spencer, R. A., Rehman, L., and Kirk, S. F. (2015) Understanding gender norms, nutrition, and physical activity in adolescent girls: a scoping review. Int J Behav Nutr Phys Act 12, 6

63. Sharara, E., Akik, C., Ghattas, H., and Makhlouf Obermeyer, C. (2018) Physical inactivity, gender and culture in Arab countries: a systematic assessment of the literature. BMC Public Health 18, 639

64. Tcymbal, A., Whiting, Mikkelsen, Rakovac, Breda. (2020) Prevalence of Physical Inactivity and Sedentary Behavior Among Adults in Armenia. Front Public Health

65. Venn, S., Arber, S., Meadows, R. and Hislop, J. (2008) The fourth shift: exploring the gendered nature of sleep disruption among couples with children. The British Journal of Sociology

66. Harry-Hernandez, S., Reisner, S. L., Schrimshaw, E. W., Radix, A., Mallick, R., Callander, D., Suarez, L., Dubin, S., Khan, A., \& Duncan, D. T. (2020) Gender Dysphoria, Mental Health, and Poor Sleep Health Among Transgender and Gender Nonbinary Individuals: A Qualitative Study in New York City. Transgender health

67. Butler, E. S., et al. . (2019) "Sexual and Gender Minority Sleep: A Narrative Review and Suggestions for Future Research.”. Journal of Sleep Research 
68. Courtenay, W. H. (2000) Constructions of masculinity and their influence on men's wellbeing: a theory of gender and health. Social Science \& Medicine

69. Courtenay, W. H. (2000) Health Factors Associated with Disease, Injury, and Death among Men: Evidence and Implications for Prevention. The Journal of Men's Studies

70. Perrotte, J. K., Shattuck, E.C., Daniels, C.L. et al. (2021) A latent profile analysis of the link between sociocultural factors and health-related risk-taking among U.S. adults. $B M C$ Public Health

71. Lee, S., Cho, E., Grodstein, F., Kawachi, I., Hu, F. B., and Colditz, G. A. (2005) Effects of marital transitions on changes in dietary and other health behaviours in US women. Int J Epidemiol 34, 69-78

72. Tian, J., Smith, K. J., Cleland, V., Gall, S., Dwyer, T., and Venn, A. J. (2020) Partnering and parenting transitions in Australian men and women: associations with changes in weight, domain-specific physical activity and sedentary behaviours. Int J Behav Nutr Phys Act 17, 87

73. Kutob, Y., Wertheim, Sbarra, Loucks, Nassir, Bareh, Kim, Snetselaar, Thomson. (2017) Relationship Between Marital Transitions, Health Behaviors, and Health Indicators of Postmenopausal Women: Results from the Women's Health Initiative. Journal of Women's Health

74. Gettler, M., Feranil, Kuzawa. (2011) Longitudinal evidence that fatherhood decreases testosterone in human males. PNAS

75. Azziz, R., Carmina, E., Chen, Z., et al. (2016) Polycystic ovary syndrome. Nature Review Disease Primers

76. Martelli, M., Zingaretti, L., Salvio, G., Bracci, M., and Santarelli, L. (2021) Influence of Work on Andropause and Menopause: A Systematic Review. Int J Environ Res Public Health 18

77. Zeeman, L., and Aranda, K. (2020) A Systematic Review of the Health and Healthcare Inequalities for People with Intersex Variance. Int J Environ Res Public Health 17

78. Rosenwohl-Mack, A., Tamar-Mattis, S., Baratz, A. B., Dalke, K. B., Ittelson, A., Zieselman, K., and Flatt, J. D. (2020) A national study on the physical and mental health of intersex adults in the U.S. PLoS One 15, e0240088

79. Fausto $\square$ Sterling, A. (2005) The Bare Bones of Sex: Part $1-$ Sex and Gender. Signs

80. Johnson, J., \& Repta, R. . (2012) Sex and gender: beyond the binaries. In Designing and conducting gender, sex, \& health research (J. L. Oliffe, L. G., ed) pp. 17 - 38, SAGE Publications

81. AlGhatrif, M., Zane, A., Oberdier, M., Canepa, M., Studenski, S., Simonsick, E., Spencer, R. G., Fishbein, K., Reiter, D., Lakatta, E. G., McDermott, M. M., and Ferrucci, 
L. (2017) Lower Mitochondrial Energy Production of the Thigh Muscles in Patients With Low-Normal Ankle-Brachial Index. J Am Heart Assoc 6, e006604

82. Arribat, Y., Broskey, N. T., Greggio, C., Boutant, M., Conde Alonso, S., Kulkarni, S. S., Lagarrigue, S., Carnero, E. A., Besson, C., Cantó, C., and Amati, F. (2019) Distinct patterns of skeletal muscle mitochondria fusion, fission and mitophagy upon duration of exercise training. Acta Physiol (Oxf) 225, e13179

83. Bañuls, C., de Marañon, A. M., Veses, S., Castro-Vega, I., López-Domènech, S., SalomVendrell, C., Orden, S., Álvarez, Á., Rocha, M., Víctor, V. M., and Hernández-Mijares, A. (2019) Malnutrition impairs mitochondrial function and leukocyte activation. Nutrition Journal 18, 89

84. Bescos, R., Boden, M. J., Jackson, M. L., Trewin, A. J., Marin, E. C., Levinger, I., Garnham, A., Hiam, D. S., Falcao-Tebas, F., Conte, F., Owens, J. A., Kennaway, D. J., and McConell, G. K. (2018) Four days of simulated shift work reduces insulin sensitivity in humans. Acta Physiol (Oxf) 223, e13039

85. Broskey, N. T., Greggio, C., Boss, A., Boutant, M., Dwyer, A., Schlueter, L., Hans, D., Gremion, G., Kreis, R., Boesch, C., Canto, C., and Amati, F. (2014) Skeletal muscle mitochondria in the elderly: effects of physical fitness and exercise training. J Clin Endocrinol Metab 99, 1852-1861

86. Cardinale, D. A., Larsen, F. J., Schiffer, T. A., Morales-Alamo, D., Ekblom, B., Calbet, J. A. L., Holmberg, H. C., and Boushel, R. (2018) Superior Intrinsic Mitochondrial Respiration in Women Than in Men. Front Physiol 9, 1133

87. Chomentowski, P., Coen, P. M., Radiková, Z., Goodpaster, B. H., and Toledo, F. G. (2011) Skeletal muscle mitochondria in insulin resistance: differences in intermyofibrillar versus subsarcolemmal subpopulations and relationship to metabolic flexibility. J Clin Endocrinol Metab 96, 494-503

88. Coggan, A. R., Spina, R. J., King, D. S., Rogers, M. A., Brown, M., Nemeth, P. M., and Holloszy, J. O. (1992) Histochemical and enzymatic comparison of the gastrocnemius muscle of young and elderly men and women. J Gerontol 47, B71-76

89. Coggan, A. R., Spina, R. J., King, D. S., Rogers, M. A., Brown, M., Nemeth, P. M., and Holloszy, J. O. (1992) Skeletal muscle adaptations to endurance training in 60- to 70-yrold men and women. J Appl Physiol (1985) 72, 1780-1786

90. Crane, J. D., Devries, M. C., Safdar, A., Hamadeh, M. J., and Tarnopolsky, M. A. (2010) The effect of aging on human skeletal muscle mitochondrial and intramyocellular lipid ultrastructure. J Gerontol A Biol Sci Med Sci 65, 119-128

91. Cree-Green, M., Cai, N., Pyle, L., Ringham, B., Brown, M. S., Newcomer, B. R., Nadeau, K. J., and Dabelea, D. (2017) Insulin Resistance in Youth Without Diabetes Is Not Related to Muscle Mitochondrial Dysfunction. J Clin Endocrinol Metab 102, 16521660 
92. Da Silva, T., Wu, A., Laksono, I., Prce, I., Maheandiran, M., Kiang, M., Andreazza, A. C., and Mizrahi, R. (2018) Mitochondrial function in individuals at clinical high risk for psychosis. Scientific Reports 8, 6216

93. DeConne, T. M., Muñoz, E. R., Sanjana, F., Hobson, J. C., and Martens, C. R. (2020) Cardiometabolic risk factors are associated with immune cell mitochondrial respiration in humans. Am J Physiol Heart Circ Physiol 319, H481-h487

94. Dodds, R. M., Davies, K., Granic, A., Hollingsworth, K. G., Warren, C., Gorman, G., Turnbull, D. M., and Sayer, A. A. (2018) Mitochondrial respiratory chain function and content are preserved in the skeletal muscle of active very old men and women. Exp Gerontol 113, 80-85

95. Dohlmann, T. L., Hindsø, M., Dela, F., Helge, J. W., and Larsen, S. (2018) Highintensity interval training changes mitochondrial respiratory capacity differently in adipose tissue and skeletal muscle. Physiol Rep 6, e13857

96. Ehinger, J. K., Morota, S., Hansson, M. J., Paul, G., and Elmér, E. (2015) Mitochondrial dysfunction in blood cells from amyotrophic lateral sclerosis patients. J Neurol 262, $1493-1503$

97. Fisher-Wellman, K. H., Weber, T. M., Cathey, B. L., Brophy, P. M., Gilliam, L. A., Kane, C. L., Maples, J. M., Gavin, T. P., Houmard, J. A., and Neufer, P. D. (2014) Mitochondrial respiratory capacity and content are normal in young insulin-resistant obese humans. Diabetes 63, 132-141

98. Gonzalez-Freire, M., Scalzo, P., D'Agostino, J., Moore, Z. A., Diaz-Ruiz, A., Fabbri, E., Zane, A., Chen, B., Becker, K. G., Lehrmann, E., Zukley, L., Chia, C. W., Tanaka, T., Coen, P. M., Bernier, M., de Cabo, R., and Ferrucci, L. (2018) Skeletal muscle ex vivo mitochondrial respiration parallels decline in vivo oxidative capacity, cardiorespiratory fitness, and muscle strength: The Baltimore Longitudinal Study of Aging. Aging Cell 17

99. Hafen, P. S., Abbott, K., Bowden, J., Lopiano, R., Hancock, C. R., and Hyldahl, R. D. (2019) Daily heat treatment maintains mitochondrial function and attenuates atrophy in human skeletal muscle subjected to immobilization. J Appl Physiol (1985) 127, 47-57

100. Hafen, P. S., Preece, C. N., Sorensen, J. R., Hancock, C. R., and Hyldahl, R. D. (2018) Repeated exposure to heat stress induces mitochondrial adaptation in human skeletal muscle. J Appl Physiol (1985) 125, 1447-1455

101. Harish, G., Venkateshappa, C., Mahadevan, A., Pruthi, N., Bharath, M. M., and Shankar, S. K. (2013) Mitochondrial function in human brains is affected by pre- and post mortem factors. Neuropathol Appl Neurobiol 39, 298-315

102. Lalia, A. Z., Dasari, S., Johnson, M. L., Robinson, M. M., Konopka, A. R., Distelmaier, K., Port, J. D., Glavin, M. T., Esponda, R. R., Nair, K. S., and Lanza, I. R. (2016) Predictors of Whole-Body Insulin Sensitivity Across Ages and Adiposity in Adult Humans. J Clin Endocrinol Metab 101, 626-634 
103. Lalia, A. Z., Dasari, S., Robinson, M. M., Abid, H., Morse, D. M., Klaus, K. A., and Lanza, I. R. (2017) Influence of omega-3 fatty acids on skeletal muscle protein metabolism and mitochondrial bioenergetics in older adults. Aging (Albany NY) 9, 10961129

104. McCully, K. K., Mulcahy, T. K., Ryan, T. E., and Zhao, Q. (2011) Skeletal muscle metabolism in individuals with spinal cord injury. J Appl Physiol (1985) 111, 143-148

105. McKeough, Z. J., Alison, J. A., Bye, P. T., Trenell, M. I., Sachinwalla, T., Thompson, C. H., and Kemp, G. J. (2006) Exercise capacity and quadriceps muscle metabolism following training in subjects with COPD. Respir Med 100, 1817-1825

106. Miller, M. S., Callahan, D. M., Tourville, T. W., Slauterbeck, J. R., Kaplan, A., Fiske, B. R., Savage, P. D., Ades, P. A., Beynnon, B. D., and Toth, M. J. (2017) Moderateintensity resistance exercise alters skeletal muscle molecular and cellular structure and function in inactive older adults with knee osteoarthritis. J Appl Physiol (1985) 122, 775787

107. Monaco, C. M. F., Hughes, M. C., Ramos, S. V., Varah, N. E., Lamberz, C., Rahman, F. A., McGlory, C., Tarnopolsky, M. A., Krause, M. P., Laham, R., Hawke, T. J., and Perry, C. G. R. (2018) Altered mitochondrial bioenergetics and ultrastructure in the skeletal muscle of young adults with type 1 diabetes. Diabetologia 61, 1411-1423

108. Panee, J., Gerschenson, M., and Chang, L. (2018) Associations Between Microbiota, Mitochondrial Function, and Cognition in Chronic Marijuana Users. J Neuroimmune Pharmacol 13, 113-122

109. Santanasto, A. J., Glynn, N. W., Jubrias, S. A., Conley, K. E., Boudreau, R. M., Amati, F., Mackey, D. C., Simonsick, E. M., Strotmeyer, E. S., Coen, P. M., Goodpaster, B. H., and Newman, A. B. (2015) Skeletal Muscle Mitochondrial Function and Fatigability in Older Adults. J Gerontol A Biol Sci Med Sci 70, 1379-1385

110. Sjövall, F., Ehinger, J. K., Marelsson, S. E., Morota, S., Frostner, E. A., Uchino, H., Lundgren, J., Arnbjörnsson, E., Hansson, M. J., Fellman, V., and Elmér, E. (2013) Mitochondrial respiration in human viable platelets--methodology and influence of gender, age and storage. Mitochondrion 13, 7-14

111. Sjövall, F., Morota, S., Hansson, M. J., Friberg, H., Gnaiger, E., and Elmér, E. (2010) Temporal increase of platelet mitochondrial respiration is negatively associated with clinical outcome in patients with sepsis. Crit Care 14, R214

112. Sjövall, F., Morota, S., Persson, J., Hansson, M. J., and Elmér, E. (2013) Patients with sepsis exhibit increased mitochondrial respiratory capacity in peripheral blood immune cells. Crit Care 17, R152

113. Smith, S. A., Montain, S. J., Zientara, G. P., and Fielding, R. A. (2004) Use of phosphocreatine kinetics to determine the influence of creatine on muscle mitochondrial respiration: an in vivo 31P-MRS study of oral creatine ingestion. J Appl Physiol (1985) 96, 2288-2292 
114. Tecilazich, F., Dinh, T., Lyons, T. E., Guest, J., Villafuerte, R. A., Sampanis, C., Gnardellis, C., Zuo, C. S., and Veves, A. (2013) Postexercise phosphocreatine recovery, an index of mitochondrial oxidative phosphorylation, is reduced in diabetic patients with lower extremity complications. J Vasc Surg 57, 997-1005

115. Tevald, M. A., Foulis, S. A., and Kent, J. A. (2014) Effect of age on in vivo oxidative capacity in two locomotory muscles of the leg. Age (Dordr) 36, 9713

116. Thompson, J. R., Swanson, S. A., Casale, G. P., Johanning, J. M., Papoutsi, E., Koutakis, P., Miserlis, D., Zhu, Z., and Pipinos, II. (2013) Gastrocnemius mitochondrial respiration: are there any differences between men and women? J Surg Res 185, 206-211

117. Thubron, E. B., Rosa, H. S., Hodges, A., Sivaprasad, S., Francis, P. T., Pienaar, I. S., and Malik, A. N. (2019) Regional mitochondrial DNA and cell-type changes in post-mortem brains of non-diabetic Alzheimer's disease are not present in diabetic Alzheimer's disease. Sci Rep 9, 11386

118. Toledo, F. G., Menshikova, E. V., Azuma, K., Radiková, Z., Kelley, C. A., Ritov, V. B., and Kelley, D. E. (2008) Mitochondrial capacity in skeletal muscle is not stimulated by weight loss despite increases in insulin action and decreases in intramyocellular lipid content. Diabetes 57, 987-994

119. Toledo, F. G., Watkins, S., and Kelley, D. E. (2006) Changes induced by physical activity and weight loss in the morphology of intermyofibrillar mitochondria in obese men and women. J Clin Endocrinol Metab 91, 3224-3227

120. Tyrrell, D. J., Bharadwaj, M. S., Van Horn, C. G., Kritchevsky, S. B., Nicklas, B. J., and Molina, A. J. (2015) Respirometric Profiling of Muscle Mitochondria and Blood Cells Are Associated With Differences in Gait Speed Among Community-Dwelling Older Adults. J Gerontol A Biol Sci Med Sci 70, 1394-1399

121. van der Lans, A. A., Hoeks, J., Brans, B., Vijgen, G. H., Visser, M. G., Vosselman, M. J., Hansen, J., Jörgensen, J. A., Wu, J., Mottaghy, F. M., Schrauwen, P., and van Marken Lichtenbelt, W. D. (2013) Cold acclimation recruits human brown fat and increases nonshivering thermogenesis. J Clin Invest 123, 3395-3403

122. Wachsmuth, M., Hübner, A., Li, M., Madea, B., and Stoneking, M. (2016) Age-Related and Heteroplasmy-Related Variation in Human mtDNA Copy Number. PLoS Genet 12, e1005939

123. Waters, D. L., Mullins, P. G., Qualls, C. R., Raj, D. S., Gasparovic, C., and Baumgartner, R. N. (2009) Mitochondrial function in physically active elders with sarcopenia. Mech Ageing Dev 130, 315-319

124. Willcocks, R. J., Williams, C. A., Barker, A. R., Fulford, J., and Armstrong, N. (2010) Age- and sex-related differences in muscle phosphocreatine and oxygenation kinetics during high-intensity exercise in adolescents and adults. NMR Biomed 23, 569-577 
125. Wu, A., Da Silva, T., Jacobson, M., Tagore, A., Lalang, N., Kiang, M., Mizrahi, R., and Andreazza, A. C. (2019) Validating mitochondrial electron transport chain content in individuals at clinical high risk for psychosis. Sci Rep 9, 12695

126. Yen, H. H., Shih, K. L., Lin, T. T., Su, W. W., Soon, M. S., and Liu, C. S. (2012) Decreased mitochondrial deoxyribonucleic acid and increased oxidative damage in chronic hepatitis C. World J Gastroenterol 18, 5084-5089

127. Yin, X., Lanza, I. R., Swain, J. M., Sarr, M. G., Nair, K. S., and Jensen, M. D. (2014) Adipocyte mitochondrial function is reduced in human obesity independent of fat cell size. J Clin Endocrinol Metab 99, E209-216 


\section{Figure legends}

\section{Figure 1 - Mitochondrial biology encompasses a broad range of functions and characteristics.}

(a) Examples of biological domains observed in humans and mitochondria. (b) Mitochondrial perform several functions, and exhibit features that inform their overall phenotypes. Abbreviated taxonomy of mitochondrial biological domains examined in this paper. $\mathrm{n}=$ total number of measures across all included papers. CI - complex I, NADH dehydrogenase; CII - complex II, succinate dehydrogenase, CIII - complex III, ubiquinol-cytochrome c reductase; CIV - complex IV, cytochrome c oxidase; CV - complex V, ATP synthase; CS, citrate synthase; mtDNAcn, mitochondrial DNA copy number; MRS, magnetic resonance spectroscopy; $\mathrm{PCr}$, phosphocreatine; ROS, reactive oxygen species

\section{Figure 2 - PRISMA flowchart of study selection process.}

Preferred reporting strategies for systematic reviews and meta-analyses (PRISMA) guideline flowchart of study eligibility and study selection. A full list of eligible articles is included in the first tab of Supplemental file 2. ${ }^{\dagger}$ Studies were considered to have too few participants when the $n$ for women or men was $<3$. ${ }^{\ddagger}$ Studies were considered to have an uneven sex ratio when there were over three times as many participants of one binary sex as the other (e.g., 30 men and 2 women). These studies were excluded because hugely unequal groups can produce unreliable effect size measures. ${ }^{\S}$ Studies were considered unavailable when we 1) failed to establish contact with the author or 2) the author was not able to access sex-disaggregated data.

\section{Figure 3 - Mitochondrial content is consistently higher in women, but OxPhos complex} protein abundance shows no consistent sex difference.

(a) Forest plots of standardized mean difference (Hedge's g) and pooled overall effect estimate $(\square)$ for mitochondrial content (CS activity unless otherwise noted), color-coded by tissue. Values $>0$ indicate higher average production in women, and values $<0$ indicate higher production in men. Study $\mathrm{n}$ (women and men combined) is noted in the table to the right of the plot. (b) Tissue-specific average effect size for mitochondrial content; number of measures (papers combined) are noted on the left. (c) Standardized mean difference (Hedge's g) and pooled effect estimate for CS activity before and after exercise training. (d) Hedge's g, complexspecific average estimate (?), and pooled effect estimate $(\downarrow)$ for oxidative phosphorylation (OxPhos) protein abundance, ordered by complex. (e) Tissue-specific average effect size for OxPhos protein abundance (all complexes combined). (f) OxPhos complex protein abundance in sedentary individuals (baseline and post exercise intervention) compared to regularly active individuals. 
Figure 4 - Neither oxidative phosphorylation enzyme activity nor in vivo MRS measures demonstrate consistent sex differences.

(a) Forest plots of standardized mean difference (Hedge's g), oxidative phosphorylation

(OxPhos) complex-specific average estimate (?), and pooled overall effect estimate $(\square)$ for OxPhos enzymatic activity, ordered by complex and color coded by tissue. Values $>0$ indicate higher average production in women, and values $<0$ indicate higher production in men. Study $n$ (women and men combined) is noted in the table to the right of the plot. (b) Tissue-specific average effect size for OxPhos enzyme activity; number of measures (papers combined) are noted on the left. (c) Hedge's g for CII activity before and after exercise training. (d) Hedge's g, measure-specific average estimate (?), and pooled effect estimate $(\bullet$ ) for in vivo MRS, ordered by measurement. (e) In vivo MRS measures in sedentary individuals (baseline and post exercise intervention), compared to themselves and/or to regularly active individuals.

\section{Figure 5 - ROS production is consistently higher in men's skeletal muscle}

(a) Forest plot of standardized mean difference (Hedge's g), substrate-specific average estimate (?), and pooled effect estimate $(\square)$ for ROS production, color-coded by tissue and ordered by substrate (oligomycin, rotenone, succinate, and assorted carbohydrate and lipid). Values $>0$ indicate higher average production in women, and values $<0$ indicate higher production in men. Study n (women and men combined) is noted in the table to the right of the plot. (b) Tissuespecific averages for ROS production; number of measures (papers combined) are noted on the left.

Figure 6 - Sex differences in phosphorylating respiration are sensitive to tissue and methodology.

Forest plots of standardized mean difference (Hedge's g) for phosphorylating mitochondrial respiration normalized to (a) CS, (b) cell, or (c) mass, ordered by methodological approach (permeabilized cells/fibers vs. isolated mitochondria) and OxPhos complex, and color-coded by tissue. Including oxidative phosphorylation (OxPhos) complex-specific average estimates (?), method-specific estimates (?), and pooled overall effect estimate $(\bullet)$. Values $>0$ indicate higher average production in women, and values $<0$ indicate higher production in men. Study $n$ (women and men combined) is noted in the table to the right of the plot. (d) Tissue-specific average effect sizes for phosphorylating respiration, normalized to mass, CS, or cell. Number of measures noted to the left of the plot.

\section{Figure 7 - Sex differences in mtDNAcn are tissue-specific.}

(a) Forest plot of standardized mean difference (Hedge's g) and pooled overall effect estimate $(\square)$ for mitochondrial DNA copy number (mtDNAcn), color-coded by tissue. Values $>0$ indicate higher average production in women, and values $<0$ indicate higher production in men. 
Study n (women and men combined) is noted in the table to the right of the plot. (b) Tissuespecific average effect sizes for mtDNAcn; number of measures is noted in the table to the left of the plot.

Figure 8 - Skeletal muscle mitochondrial volume density and size lack consistent sex differences.

(a) Forest plot of standardized mean difference (Hedge's g) and pooled overall effect estimate $(\downarrow)$ for mitochondrial volume density. Values $>0$ indicate higher average production in women, and values $<0$ indicate higher production in men. Study $n$ (women and men combined) is noted in the table to the right of the plot. (b) Measures of mitochondrial volume density in sedentary individuals (baseline and post exercise intervention), compared to themselves and/or to regularly active individuals. (c) Hedge's g and pooled effect estimate $(\square)$ for mitochondrial size. (d) (Measures of mitochondrial size in sedentary individuals (baseline and post exercise intervention)

Figure 9 - Binary sex differences in mitochondrial biology show weak to no association with age and BMI.

(a) Correlation (Spearman r) between mitochondrial sex differences and average group age per measure, color-coded by tissue. The size and color of the circle represent the slope and strength of the correlation. Significantly significant correlations $(p<0.05)$ are noted with an asterisk. Sample size is provided in terms of the number of studies, measures, and individuals. (b) Spearman $r$ between mitochondrial sex differences and average BMI per measure, with points color-coded by tissue.

Figure 10 - Observed sex differences in mitochondrial biology are negatively correlated with sample size.

(a) Scatterplots showing the relationship between effect size and sample size by domain of mitochondrial biology. Each point is a measure from a study, previously represented by a row on a forest plot; measures $>0$ indicate higher values in women, and measures $<0$ indicate higher values in men. Presented correlations (Spearman r) and p-values were found by running correlations between the sample size and the absolute value of the effect size. (b) Scatterplot showing the relationship and Spearman's $r$ between sample size and effect size for all included measures.

\section{Supplemental figure 1 - Leak/State 4 respiration does not demonstrate consistent binary sex differences.}


Forest plots of standardized mean difference (Hedge's g) for nonphosphorylating mitochondrial respiration normalized to (a) CS, (b) cell, or (c) mass, ordered by methodological approach (permeabilized cells/fibers vs. isolated mitochondria) and OxPhos complex, and color-coded by tissue. Including oxidative phosphorylation (OxPhos) complex-specific average estimates (?), method-specific estimates (?), and pooled overall effect estimate $(\bullet)$. Values $>0$ indicate higher average production in women, and values $<0$ indicate higher production in men. Study $n$ (women and men combined) is noted in the table to the right of the plot. (d) Tissue-specific average effect sizes for phosphorylating respiration, normalized to mass, CS, or cell. Number of measures noted to the left of the plot. Nonphosphorylating respiration normalized to (e) CS or (f) mass in sedentary individuals (baseline and post exercise intervention). (g) Hedges g and pooled overall effect estimate $(\bullet)$ for basal and maximal respiration in combined PBMCs.

\section{Supplemental file 1 - Data template}

\section{Supplemental file 2 - Data log}


a

\section{Domains of human health}

\section{Domains of mitochondrial biology}

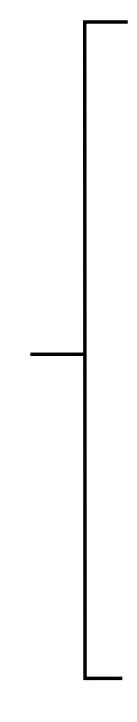

$\begin{aligned} \text { Growth } & \text { Respiration (OxPhos) } \\ \text { Physical activity } & \text { ROS emission } \\ \text { Recovery } & \text { Content } \\ \text { Sleep } & \text { Morphology } \\ \text { Digestion } & \text { Dynamics } \\ \text { Social interaction } & \text { Hormone synthesis } \\ \text { Learning } & \text { Others... }\end{aligned}$

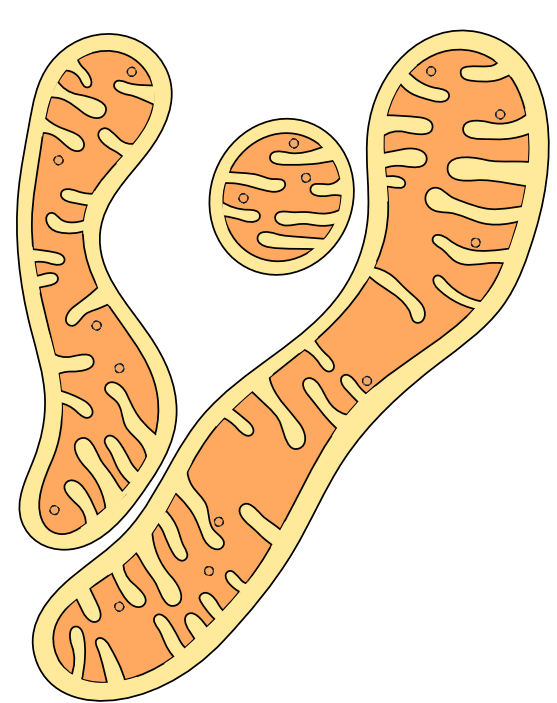

b

Mitochondrial biology

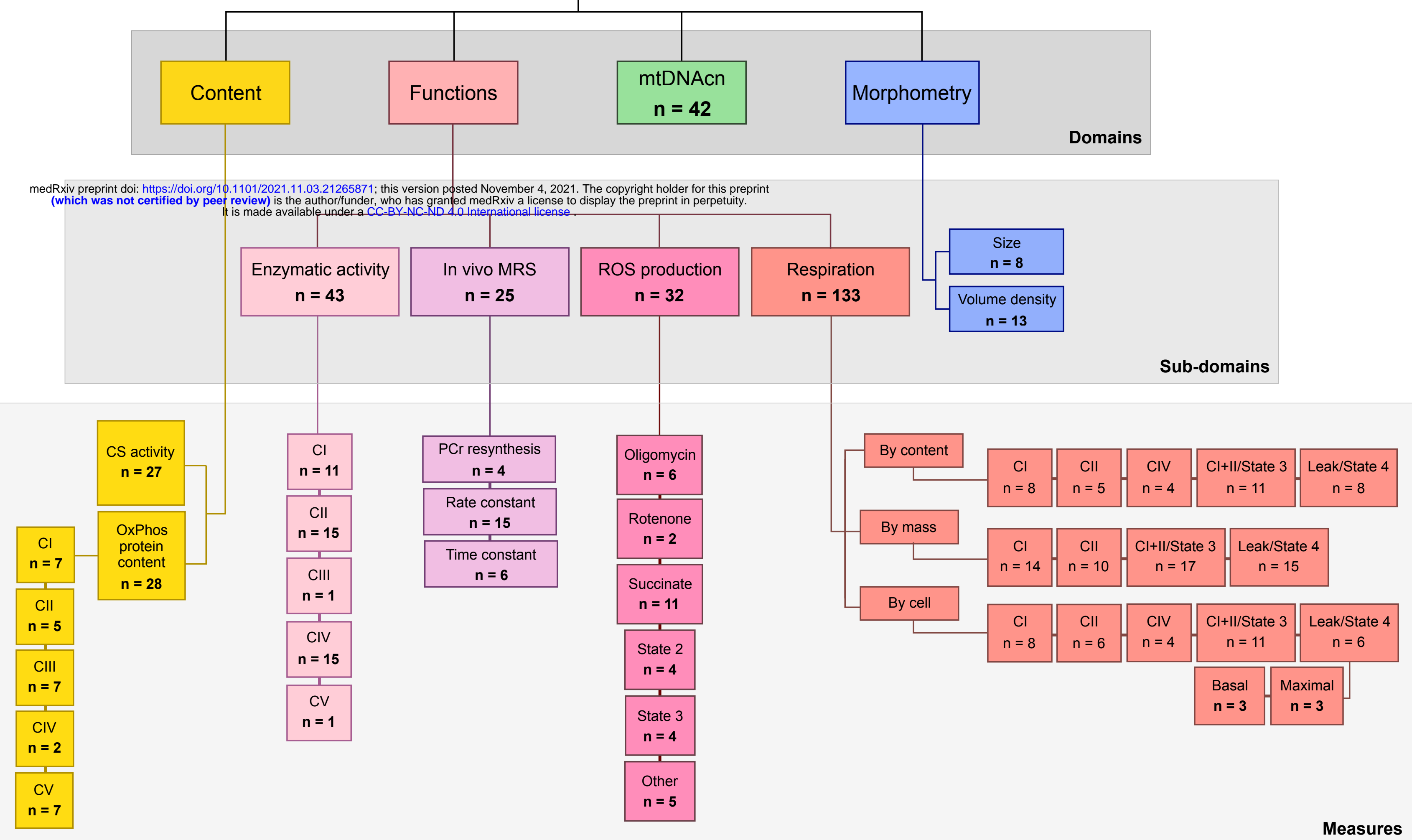

Figure 1 - Mitochondrial biology encompasses a broad range of functions and characteristics.

(a) Examples of biological domains observed in humans and mitochondria. (b) Mitochondrial perform several functions, and exhibit features that inform their overall phenotypes. Abbreviated taxonomy of mitochondrial biological domains examined in this paper. $\mathrm{n}=$ total number of measures across all included papers. $\mathrm{Cl}-\mathrm{complex} \mathrm{I}, \mathrm{NADH}$ dehydrogenase; $\mathrm{CII}$ - complex II, succinate dehydrogenase, CIII - complex III, ubiquinol-cytochrome c reductase; CIV - complex IV, cytochrome c oxidase; CV - complex V, ATP synthase; CS, citrate synthase; mtDNAcn, mitochondrial DNA copy number; MRS, magnetic resonance spectroscopy; PCr, phosphocreatine; ROS, reactive oxygen species 
Figure 2

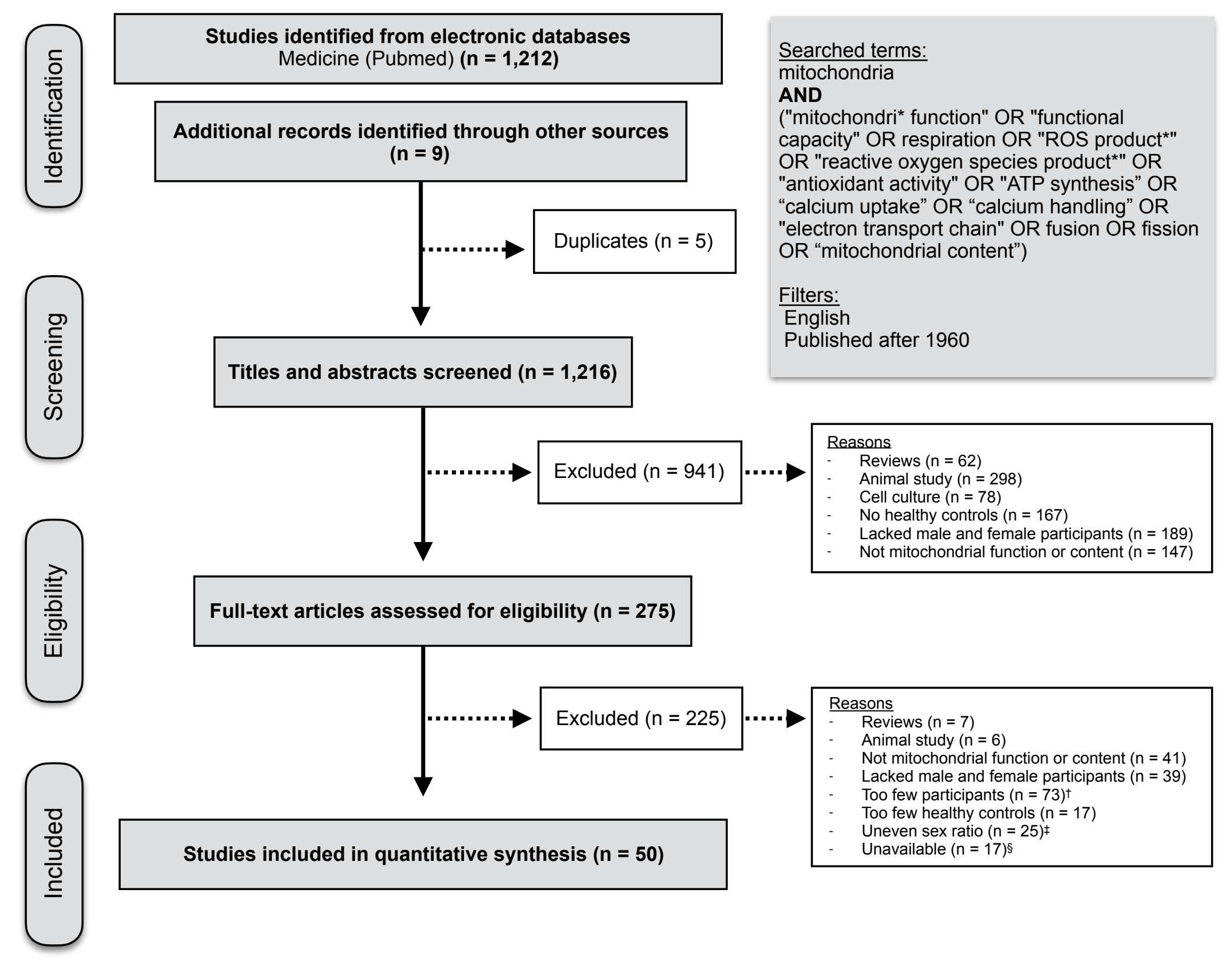

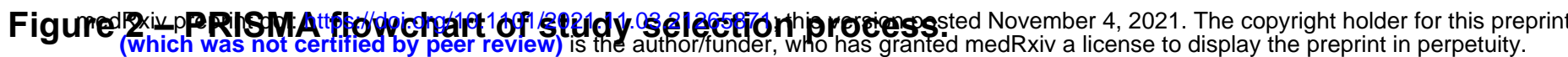

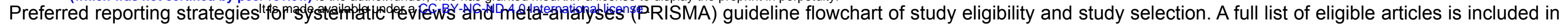
the first tab of Supplemental file 2. tStudies were considered to have too few participants when the $\mathrm{n}$ for women or men was $<3$. $\neq$ Studies were considered to have an uneven sex ratio when there were over three times as many participants of one binary sex as the other (e.g., 30 men and 2 women). These studies were excluded because hugely unequal groups can produce unreliable effect size measures. \$Studies were considered unavailable when we 1) failed to establish contact with the author or 2) the author was not able to access sex-disaggregated data. 
Mitochondrial content

a

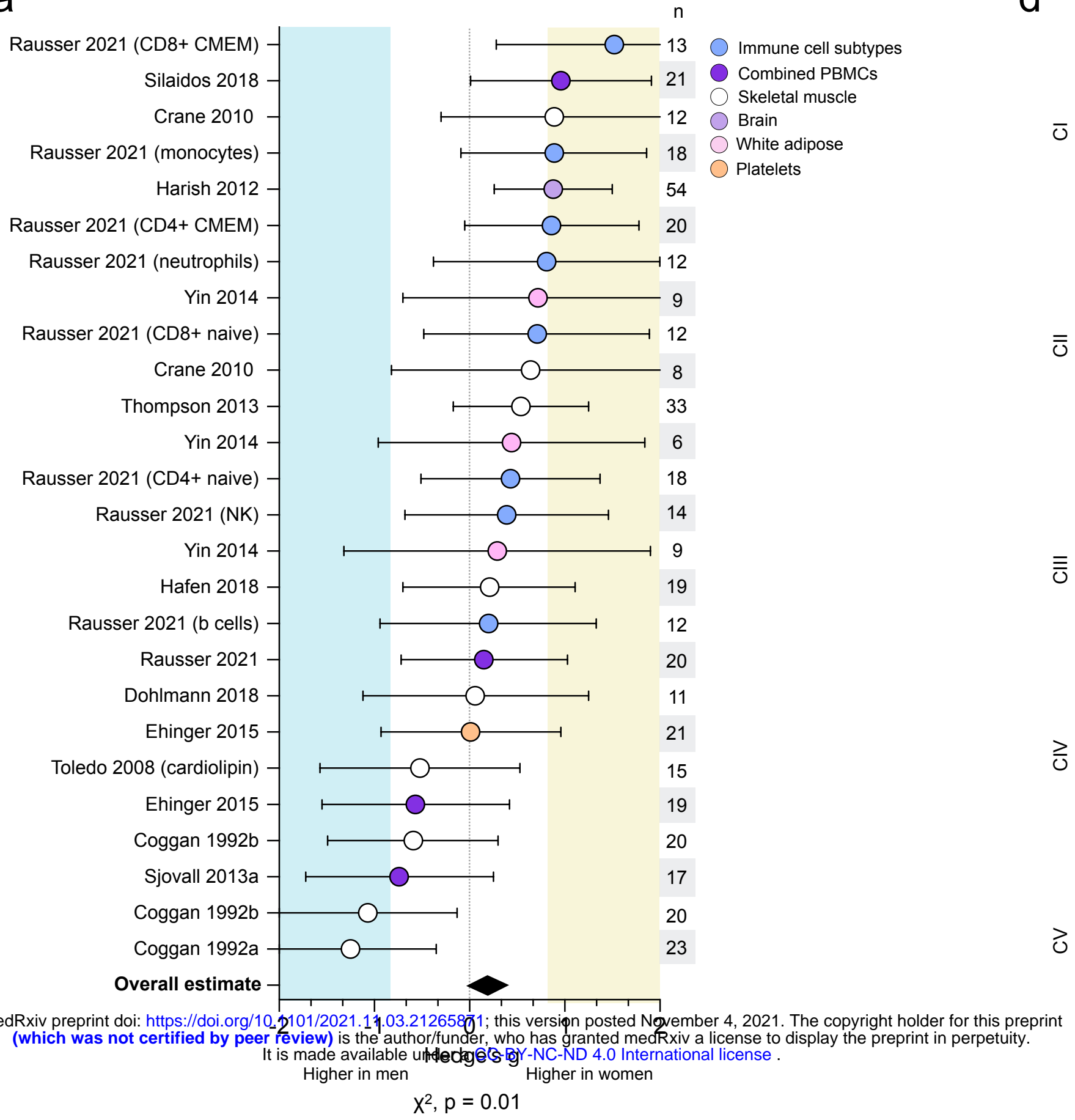

b

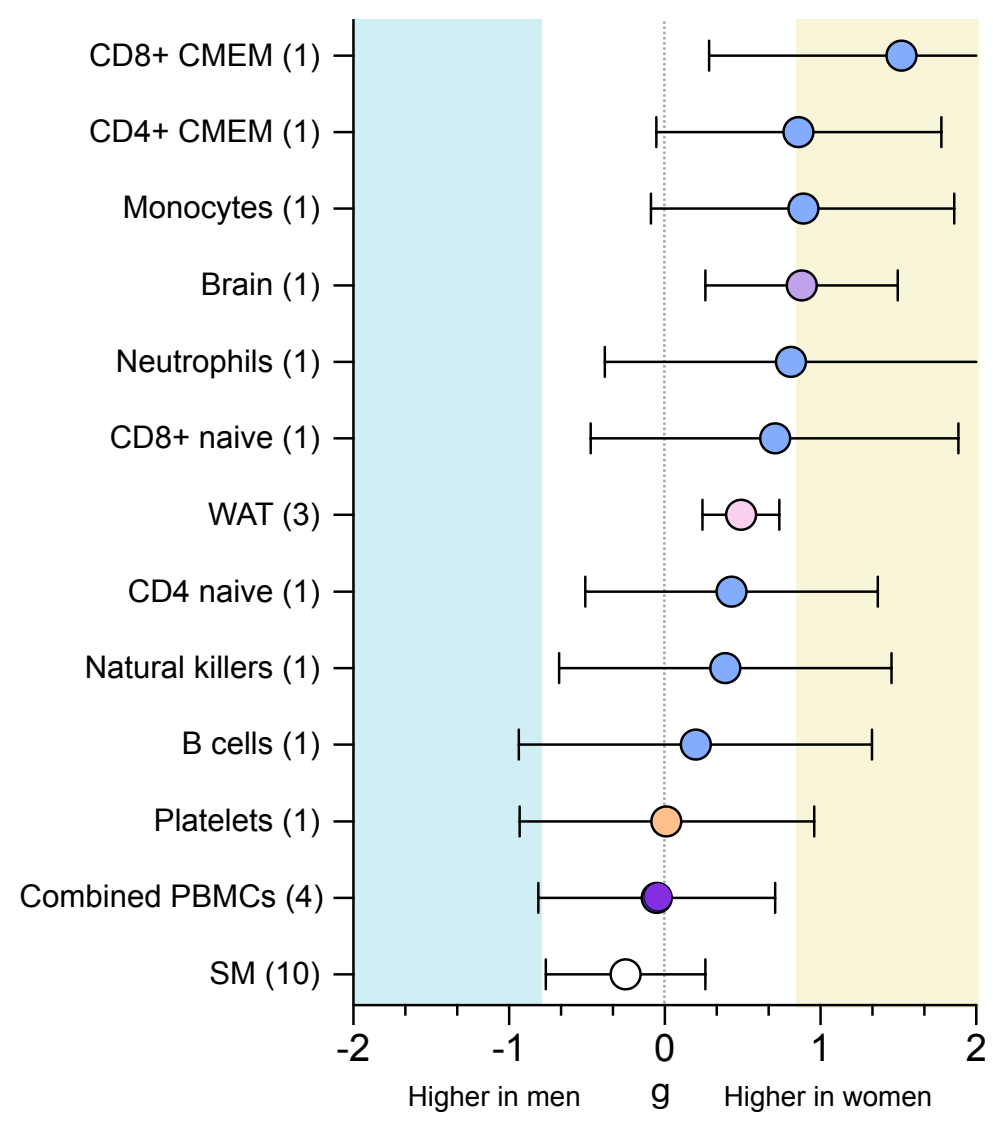

C

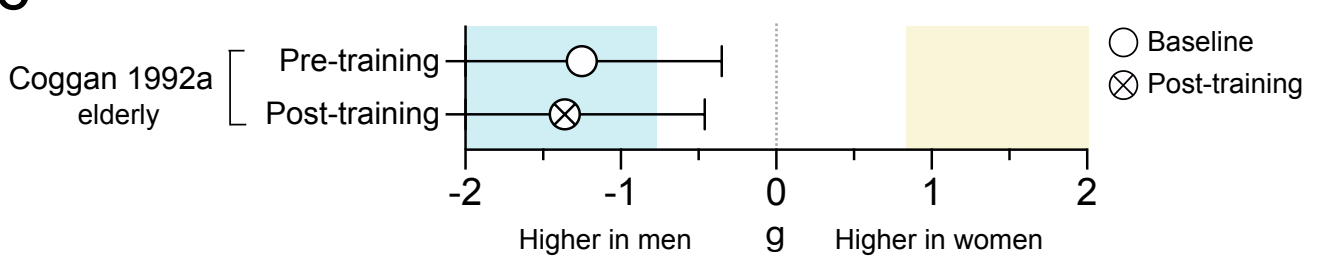

OxPhos complex protein abundance

$d$
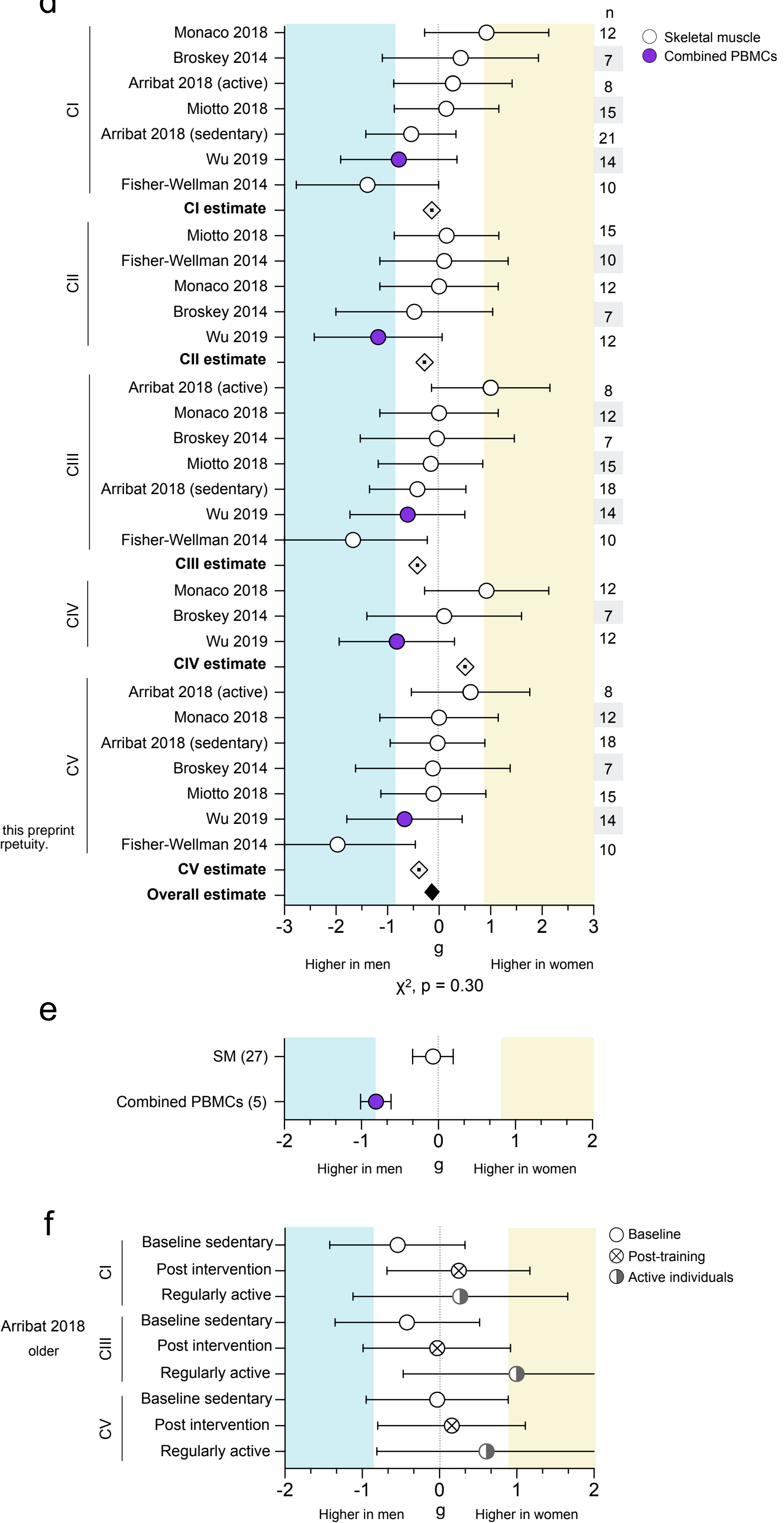

Baseline

$\otimes$ Post-training

Active individuals

Figure 3 - Mitochondrial content is consistently higher in women, but OxPhos complex protein abundance shows no consistent sex difference.

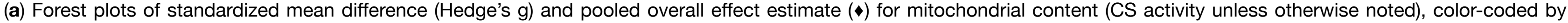

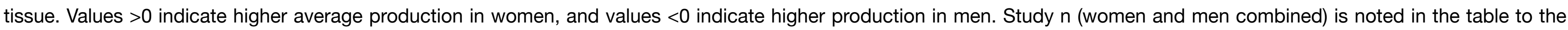

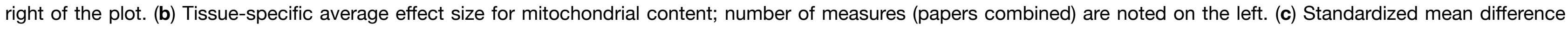

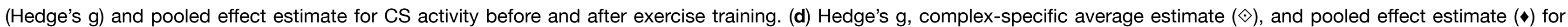

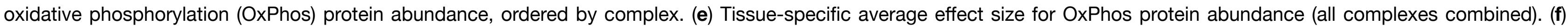
OxPhos complex protein abundance in sedentary individuals (baseline and post exercise intervention) compared to regularly active individuals. 


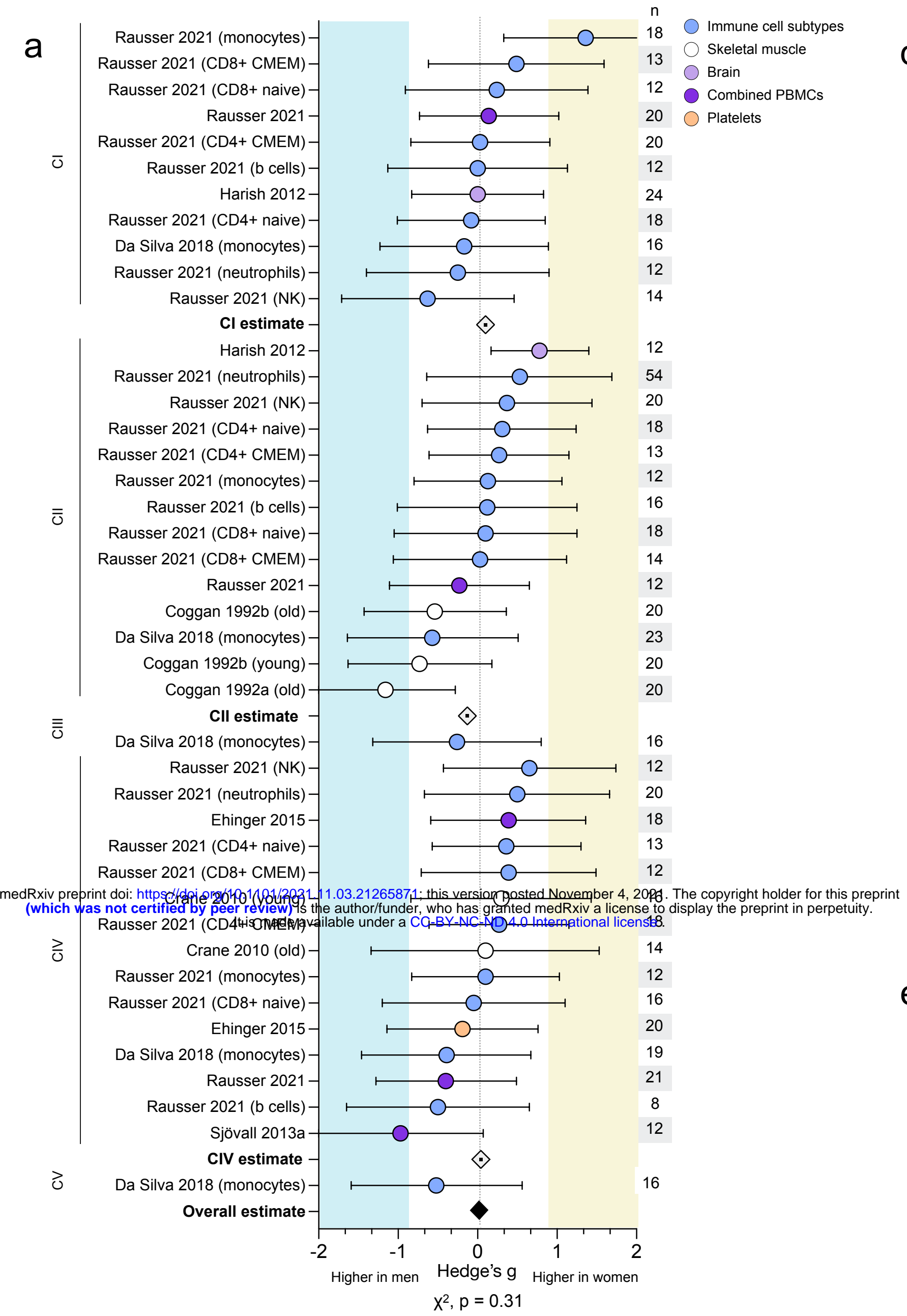

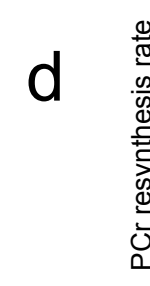

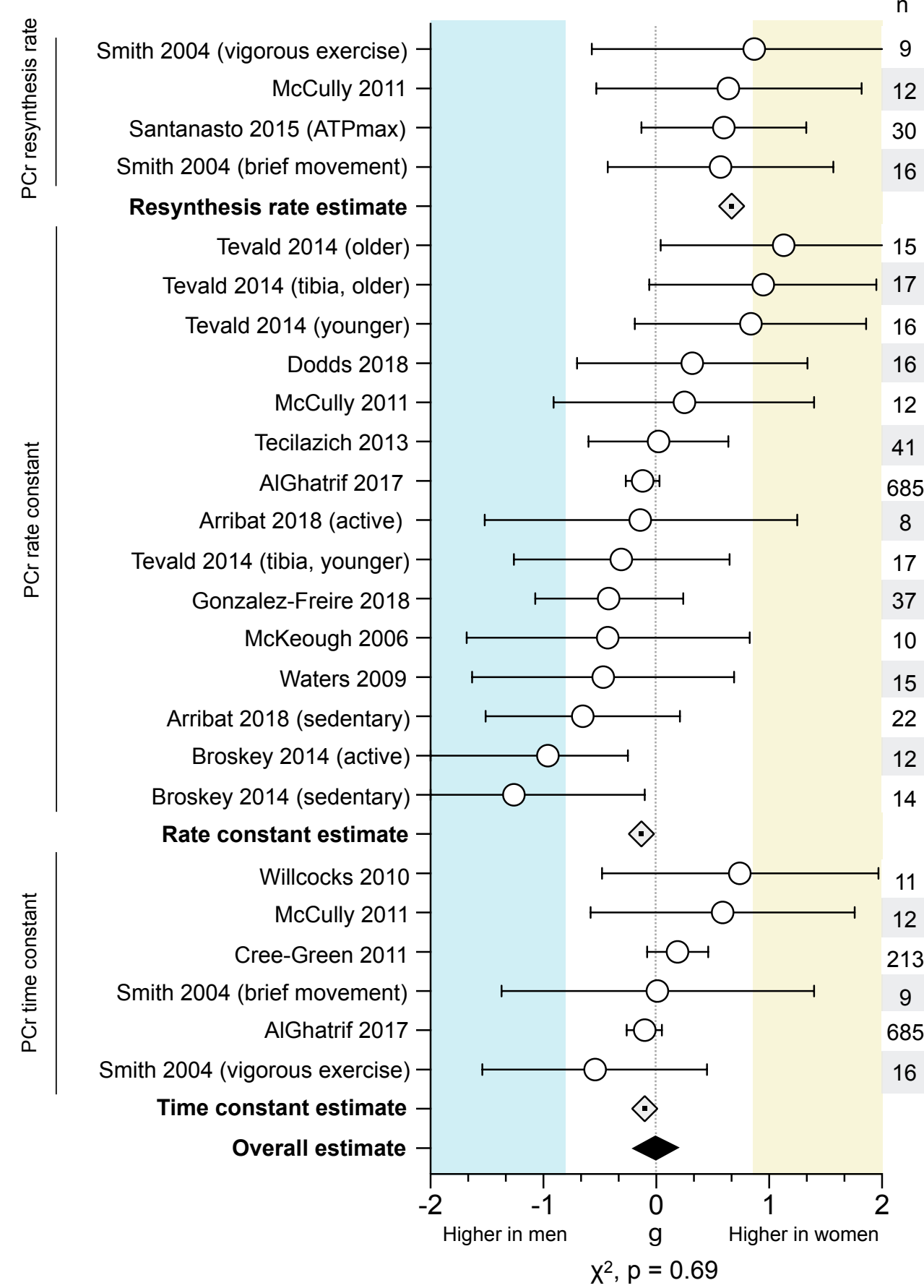

e

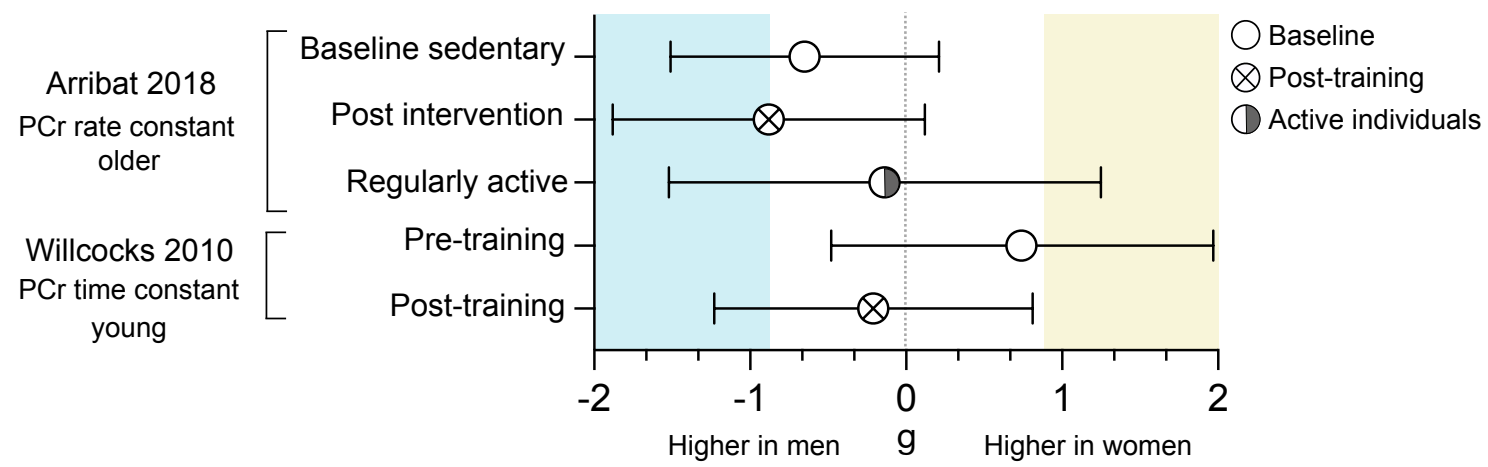

b

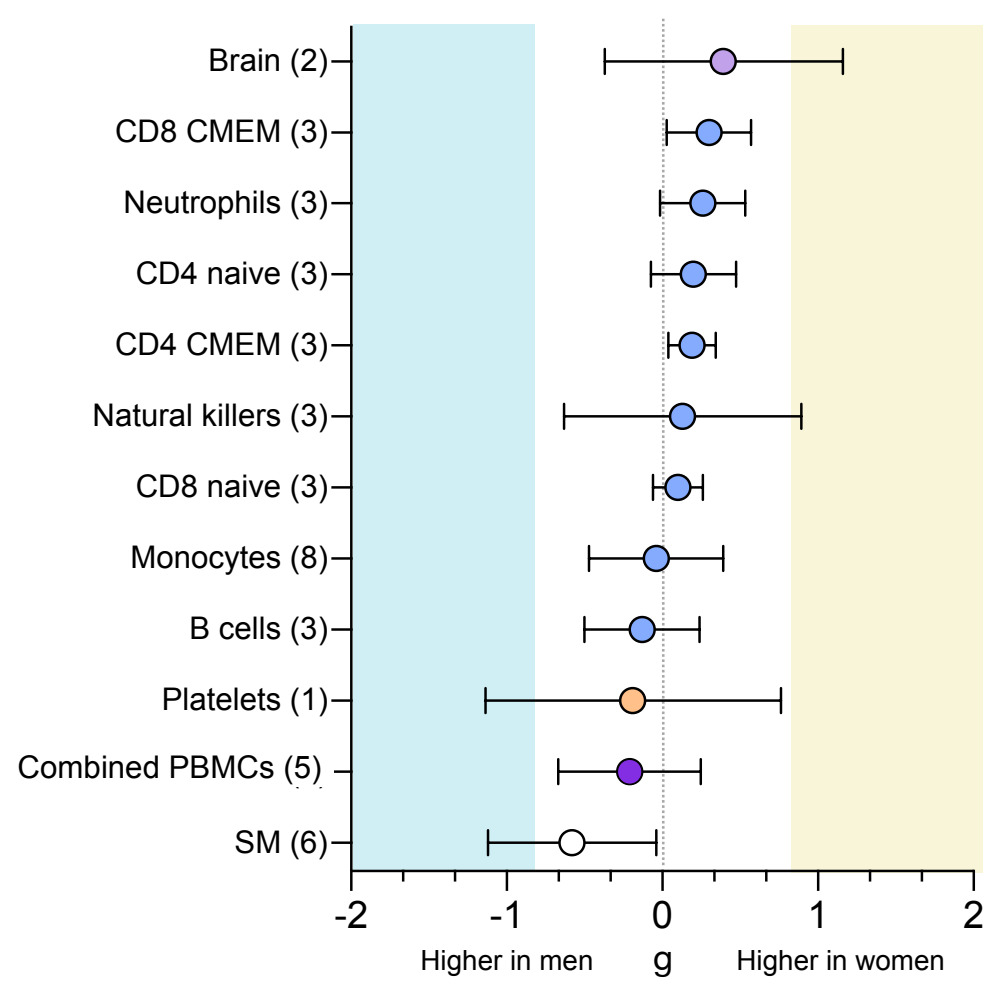

C

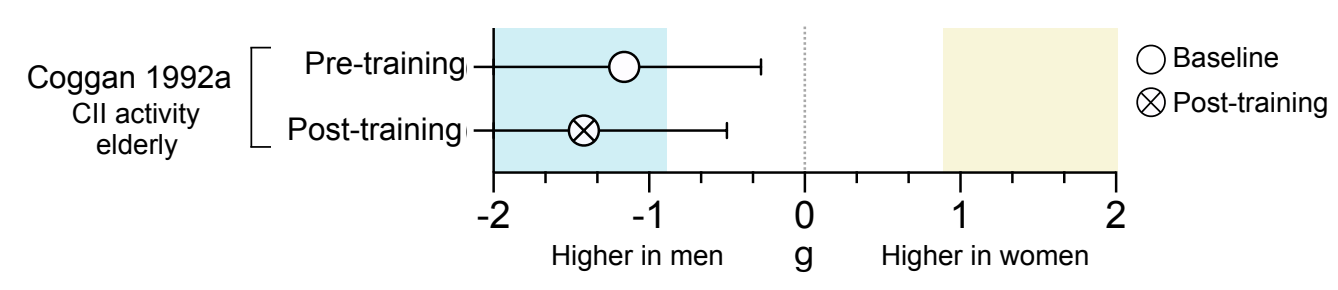

Figure 4 - Neither oxidative phosphorylation enzyme activity nor in vivo MRS measures demonstrate consistent sex differences.

(a) Forest plots of standardized mean difference (Hedge's g), oxidative phosphorylation (OxPhos) complex-specific average estimate $(\bullet)$ ), and pooled overall effect estimate $(\bullet)$ for OxPhos enzymatic activity, ordered by complex and color coded by tissue. Values $>0$ indicate higher average production in women, and values $<0$ indicate higher production in men. Study $\mathrm{n}$ (women and men combined) is noted in the table to the right of the plot. (b) Tissue-specific average effect size for OxPhos enzyme activity; number of measures (papers combined) are noted on the left. (c) Hedge's g for Cll activity before and after exercise training. (d) Hedge's g, measure-specific average estimate $(\diamond)$, and pooled effect estimate $(\diamond)$ for in vivo MRS, ordered by measurement. (e) In vivo MRS measures in sedentary individuals (baseline and post exercise intervention), compared to themselves and/or to regularly active individuals. 


\section{ROS production}

a

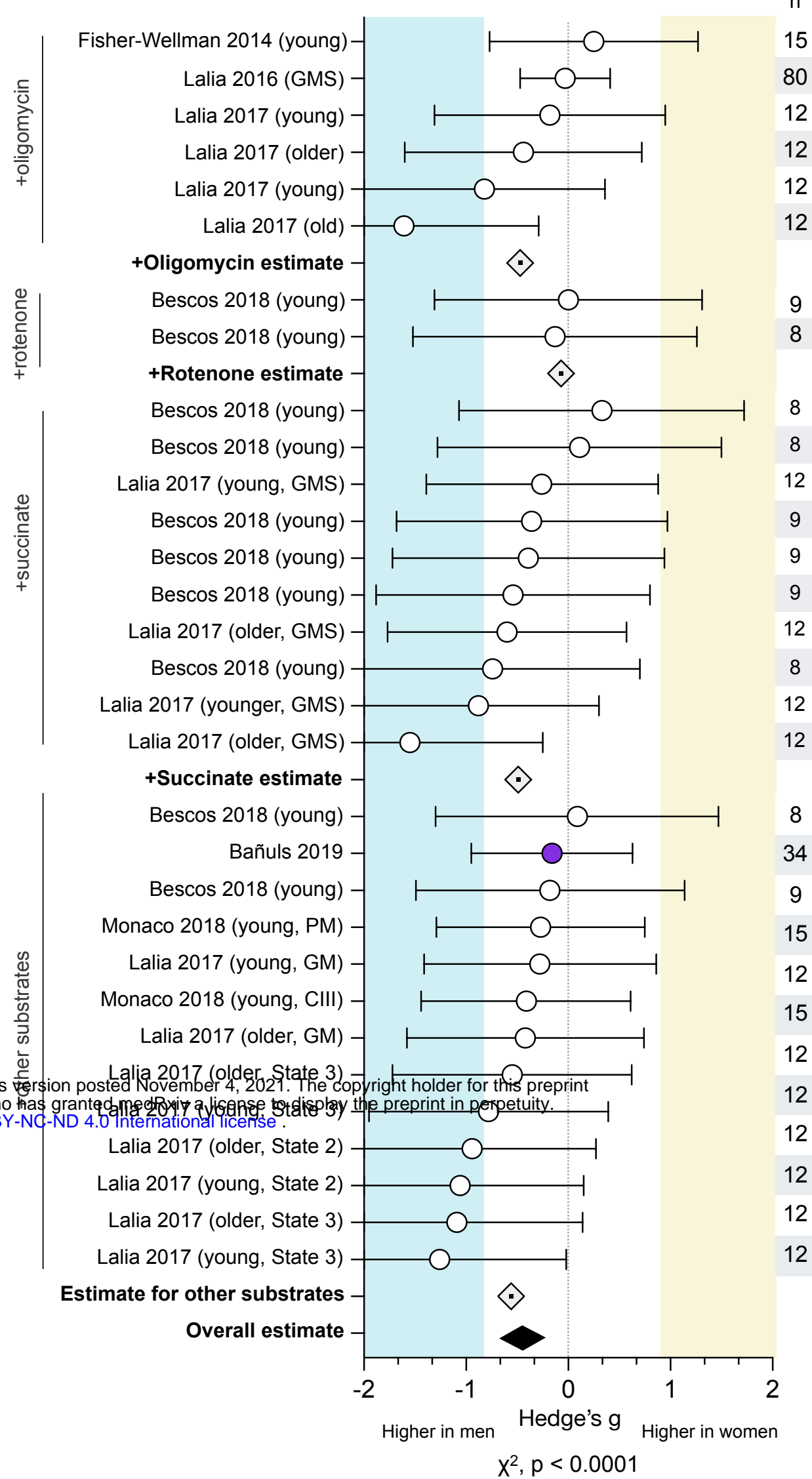

b

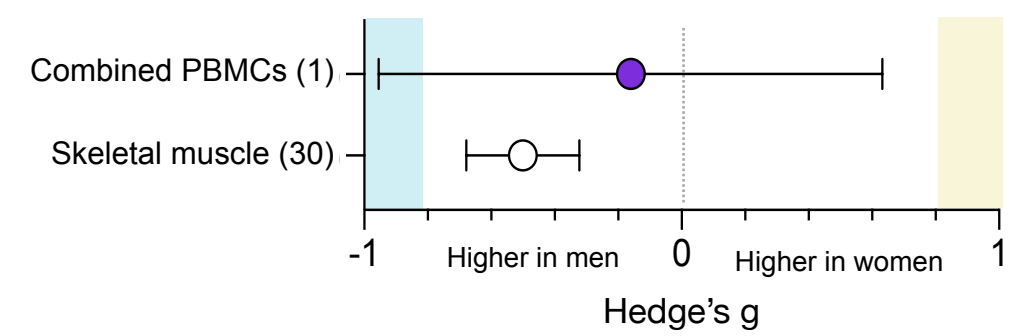

Hedge's $g$

Figure 5 - ROS production is consistently higher in men's skeletal muscle

(a) Forest plot of standardized mean difference (Hedge's g), substrate-specific average estimate $(\diamond)$ ), and pooled effect estimate ( $\bullet$ ) for ROS production, color-coded by tissue and ordered by substrate (oligomycin, rotenone, succinate, and assorted carbohydrate and lipid). Values $>0$ indicate higher average production in women, and values $<0$ indicate higher production in men. Study $\mathrm{n}$ (women and men combined) is noted in the table to the right of the plot. (b) Tissue-specific averages for ROS production; number of measures (papers combined) are noted on the left.

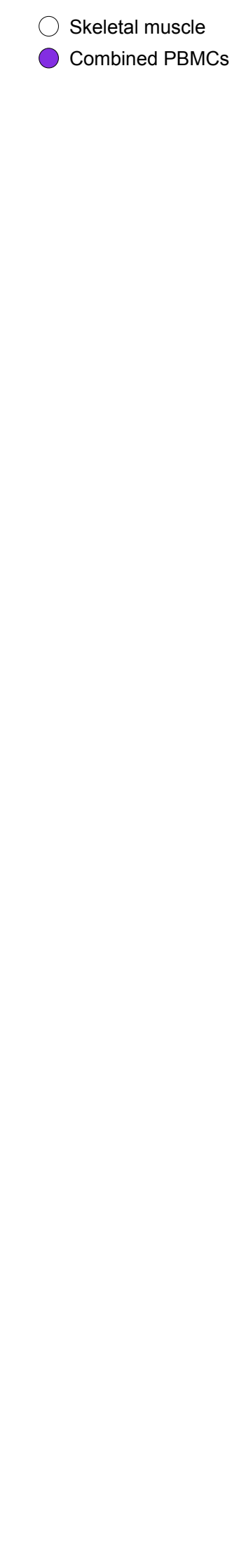


a

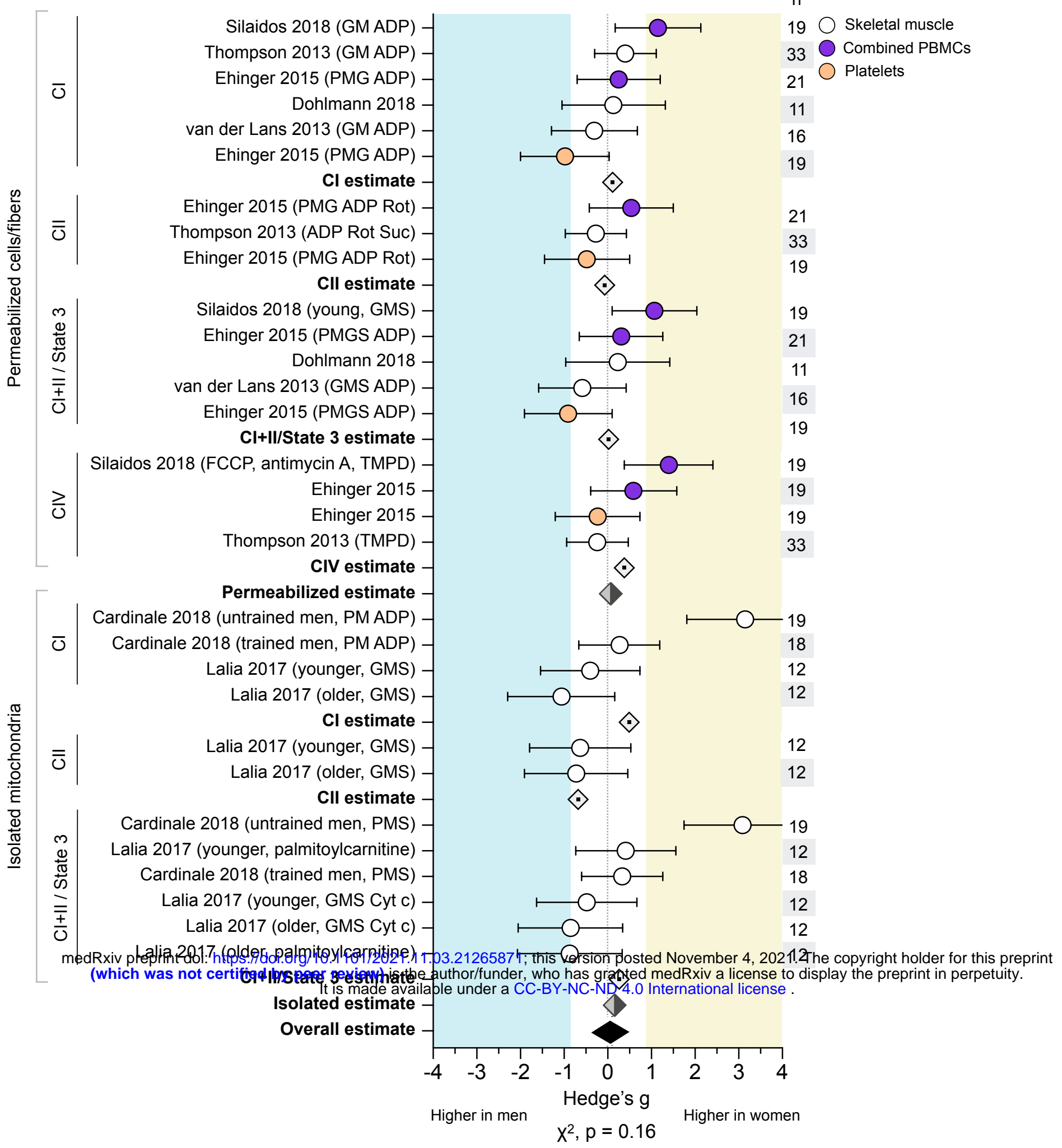

b

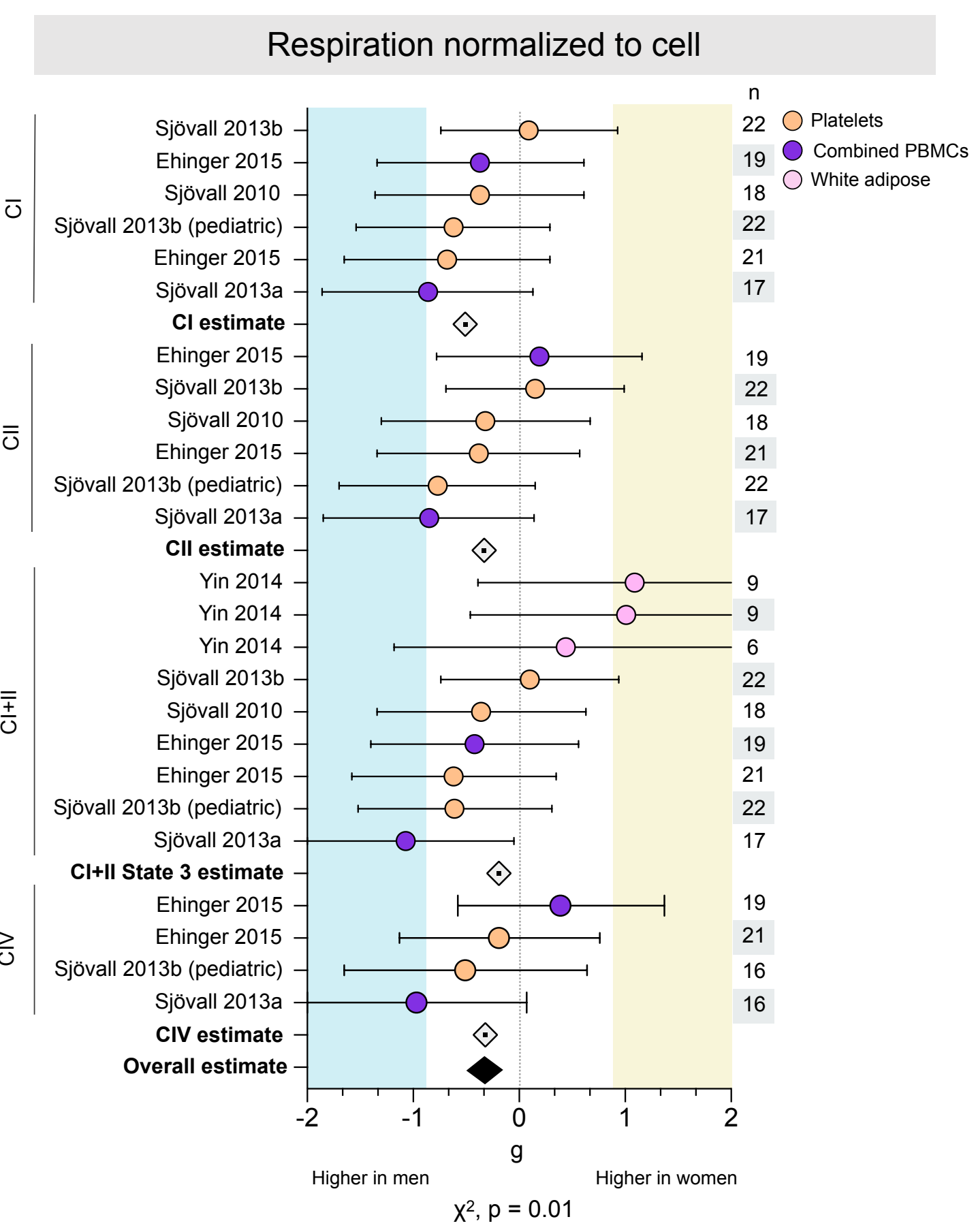

C $\overline{0}$

$\bar{u}$

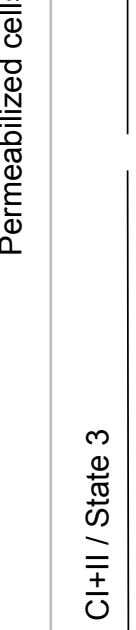

$\overline{0}$

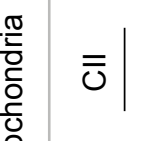

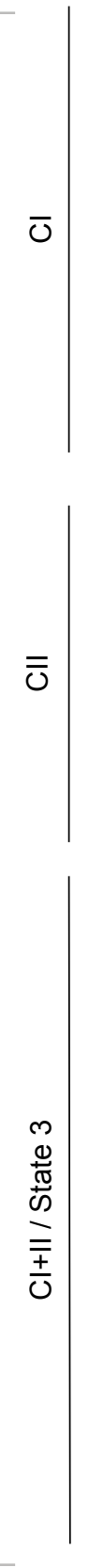

Miotto $2018(\max \mathrm{Cl})$ Miotto 2018

Monaco 2018

Dohlmann 2018

Hafen 2018

Monaco 2018

Miotto 2018

Monaco 2018

Cl estimate -

Hafen 2019

Fisher-Wellman 2014

Bescos 2018 (ETS)

Hafen 2019 (uncoupled)

Thompson 2013

Hafen 2018 (uncoupled) -

Bescos 2018 (pre-night) Monaco 2018

Cll estimate

Bescos 2018 (pre-day)

Bescos 2018 (pre-day)

Dohlmann 2018

Fisher-Wellman 2014

Hafen 2019 (uncoupled) Miotto 2018

Fisher-Wellman 2014 Hafen 2018

Fisher-Wellman 2014

Bescos 2018 (pre-night) Dohlmann 2018

Hafen 2018 (uncoupled) -

Bescos 2018 (pre-night)

Gonzalez-Frieire 2018

Fisher-Wellman 2014

Fisher-Wellman 2014

$\mathrm{Cl}+\mathrm{II} / \mathrm{State} 3$ estimate

Thompson 2013

Permeabilized estimate

Lalia 2017 (younger) Cardinale 2018 (untrained men) Cardinale 2018 (trained men)

Lalia 2017 (older)

Lalia 2017 (younger)

Lalia 2017 (older) Cll estimate -

Lalia 2017 (younger, State 3)

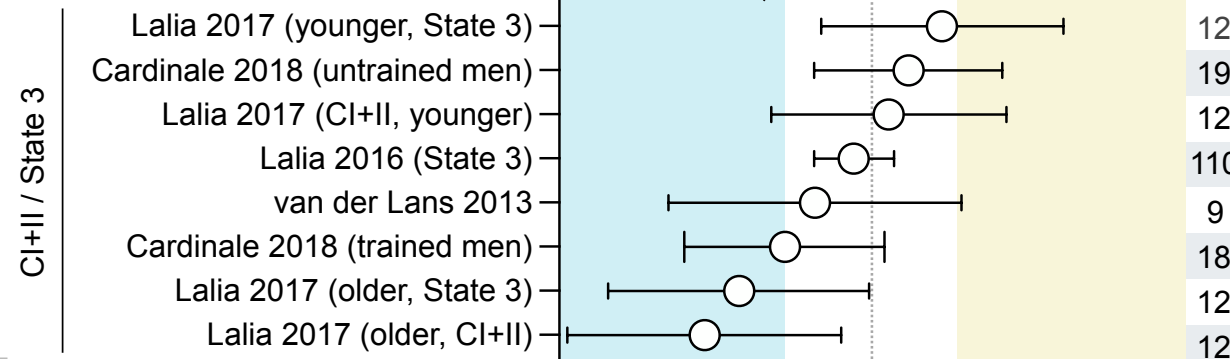

Cl+II/State 3 estimate -

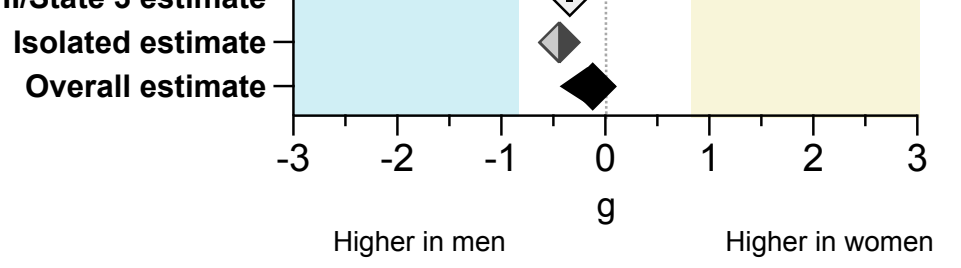

$X^{2}, p=0.56$

d

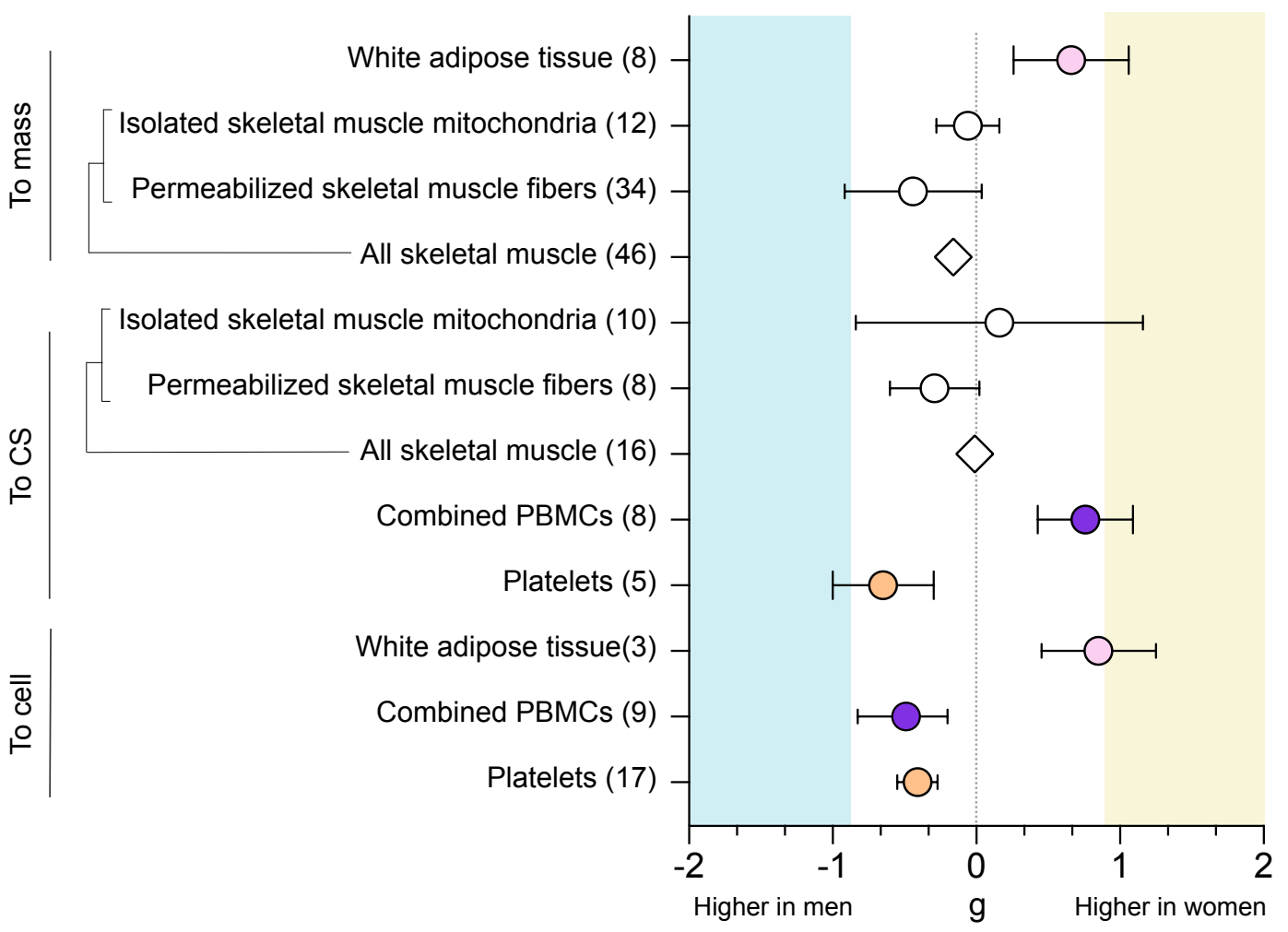

Figure 6 - Sex differences in phosphorylating respiration are sensitive to tissue and methodology.

Forest plots of standardized mean difference (Hedge's g) for phosphorylating mitochondrial respiration normalized to (a) CS, (b) cell, or (c) mass, ordered by methodological approach (permeabilized cells/fibers vs. isolated mitochondria) and OxPhos complex, and color-coded by tissue. Including oxidative phosphorylation (OxPhos) complex-specific average estimates $(\odot)$, method-specific estimates $(\diamond)$, and pooled overall effect estimate $(\triangleleft)$. Values $>0$ indicate higher average production in women, and values $<0$ indicate higher production in men. Study $\mathrm{n}$ (women and men combined) is noted in the table to the right of the plot. (d) Tissue-specific average effect sizes for phosphorylating respiration, normalized to mass, CS, or cell. Number of measures noted to the left of the plot. 


\section{Mitochondrial DNA copy number}

a

Dohlmann 2018 Rausser 2021 (CD8+ CMEM)

Rausser 2021 (b cells) Wachsmuth 2016 (shotgun MiSeq) Yin 2014

Rausser 2021 (CD4+ CMEM) Yin 2014

Wachsmuth 2016 (capture-enrichment) Wachsmuth 2016 (shotgun HiSeq) Wachsmuth 2016 (PCR) Wachsmuth 2016 (capture-enrichment) Thubron 2019 (frontal cortex) Rausser 2021 (NK) Rausser 2021 (CD4+ naive) Rausser 2021 Wachsmuth 2016 (cerebrum) van der Lans 2013 Sjovall 2010 Wachsmuth 2016

Rausser 2021 (CD8+ naive) Wachsmuth 2016 (shotgun HiSeq) Wachsmuth 2016 (capture-enrichment)

Wachsmuth 2016 (cerebellum) Wachsmuth 2016

Thubron 2019 (parietal cortex)Sjovall 2013a Yen 2012

Wachsmuth 2016 (shotgun HiSeq) Wachsmuth 2016 (PCR) Wachsmuth 2016 (capture-enrichment) Yin 2014

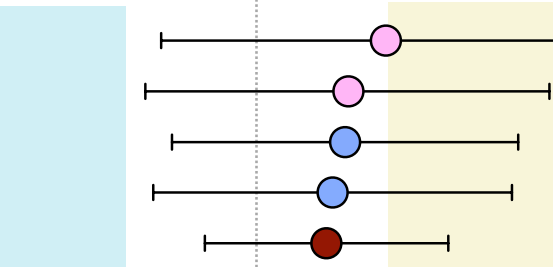

$8 \bigcirc$ White adipose

$10 \bigcirc$ Immune cell subtypes

$13 \bigcirc$ Blood

$12 \bigcirc$ Skeletal muscle

$26 \bigcirc$ Brain

Combined PBMCs

Platelets

90 Intestine

9 Liver

$139 \bigcirc$ Kidney

$148 \bigcirc$ Myocardial muscle

$150 \bigcirc$ Skin

151

16

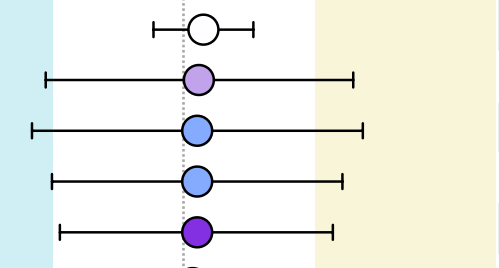

$10-1$

143

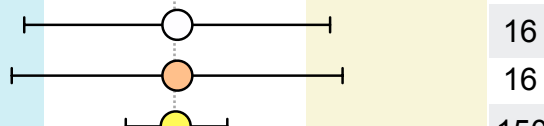

$10-1$

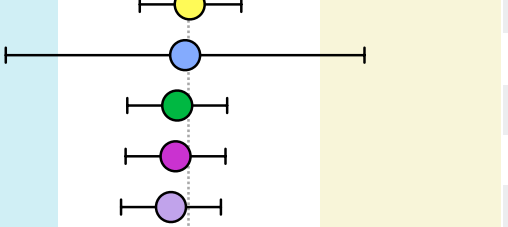

0

$10-1$

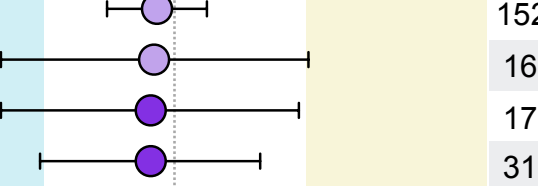

$1 \mathrm{O}-1$

$1 \mathrm{O} \quad 152$

10

$-$

10

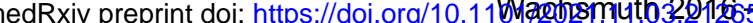

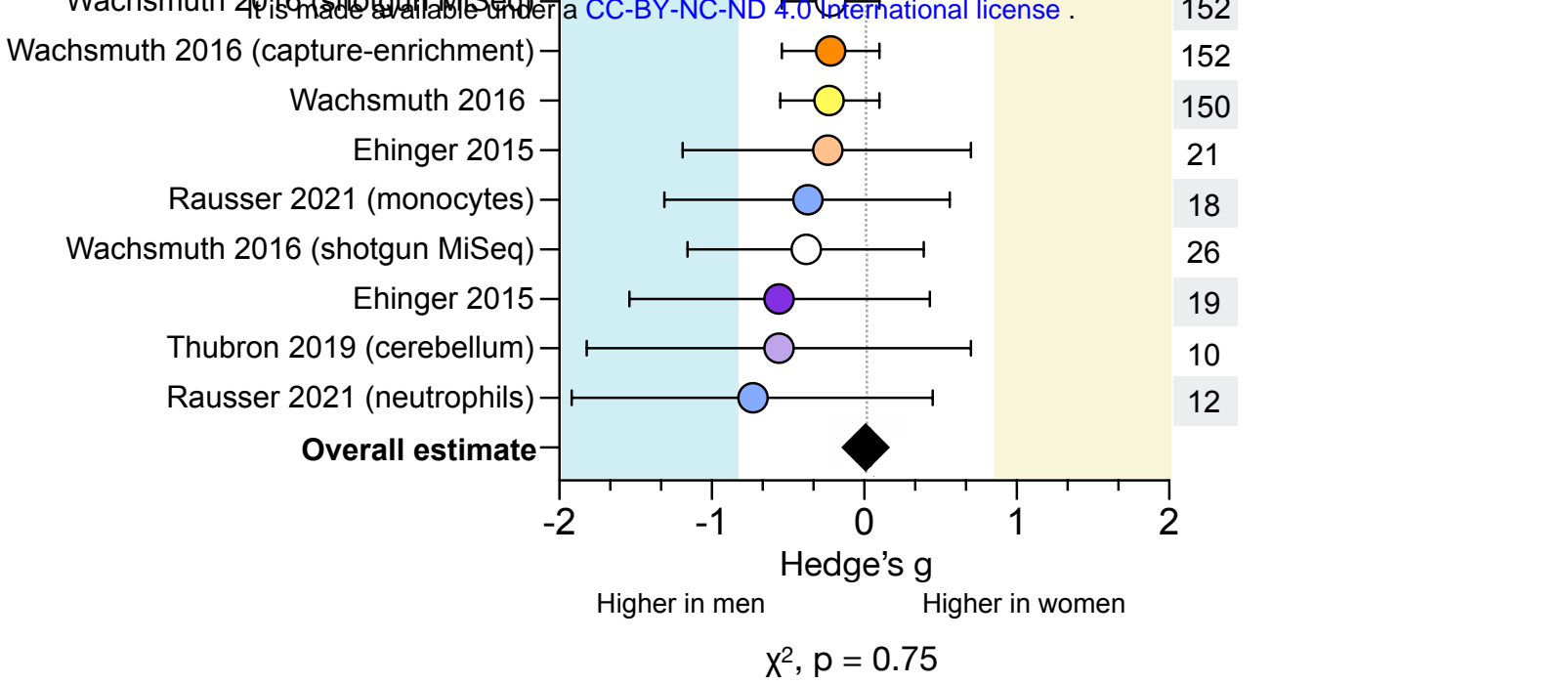

b

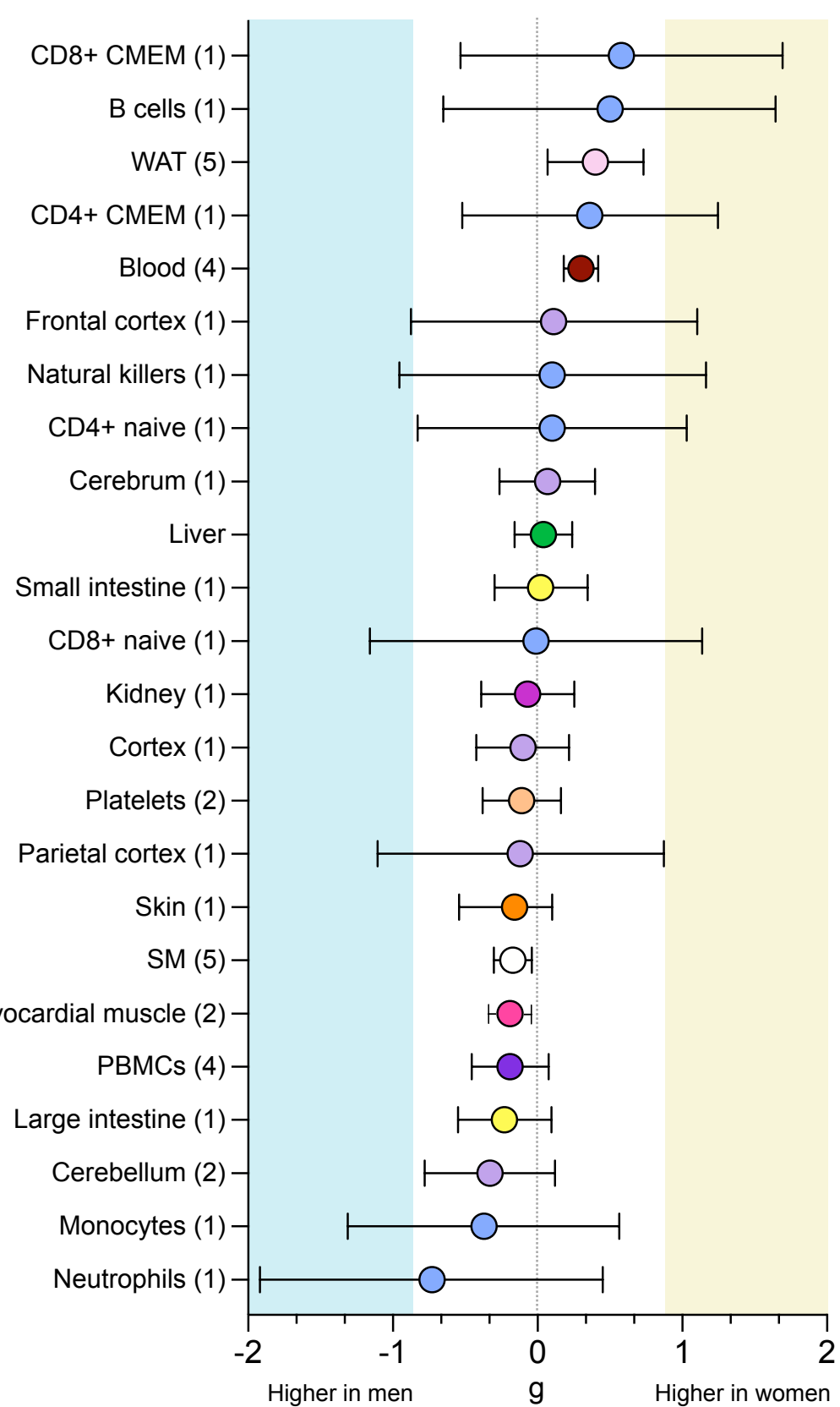

Figure 7 - Sex differences in mtDNAcn are tissue-specific.

(a) Forest plot of standardized mean difference (Hedge's g) and pooled overall effect estimate ( $\downarrow$ ) for mitochondrial DNA copy number (mtDNAcn), colorcoded by tissue. Values $>0$ indicate higher average production in women, and values $<0$ indicate higher production in men. Study $n$ (women and men combined) is noted in the table to the right of the plot. (b) Tissue-specific average effect sizes for mtDNAcn; number of measures is noted in the table to the left of the plot. 
a

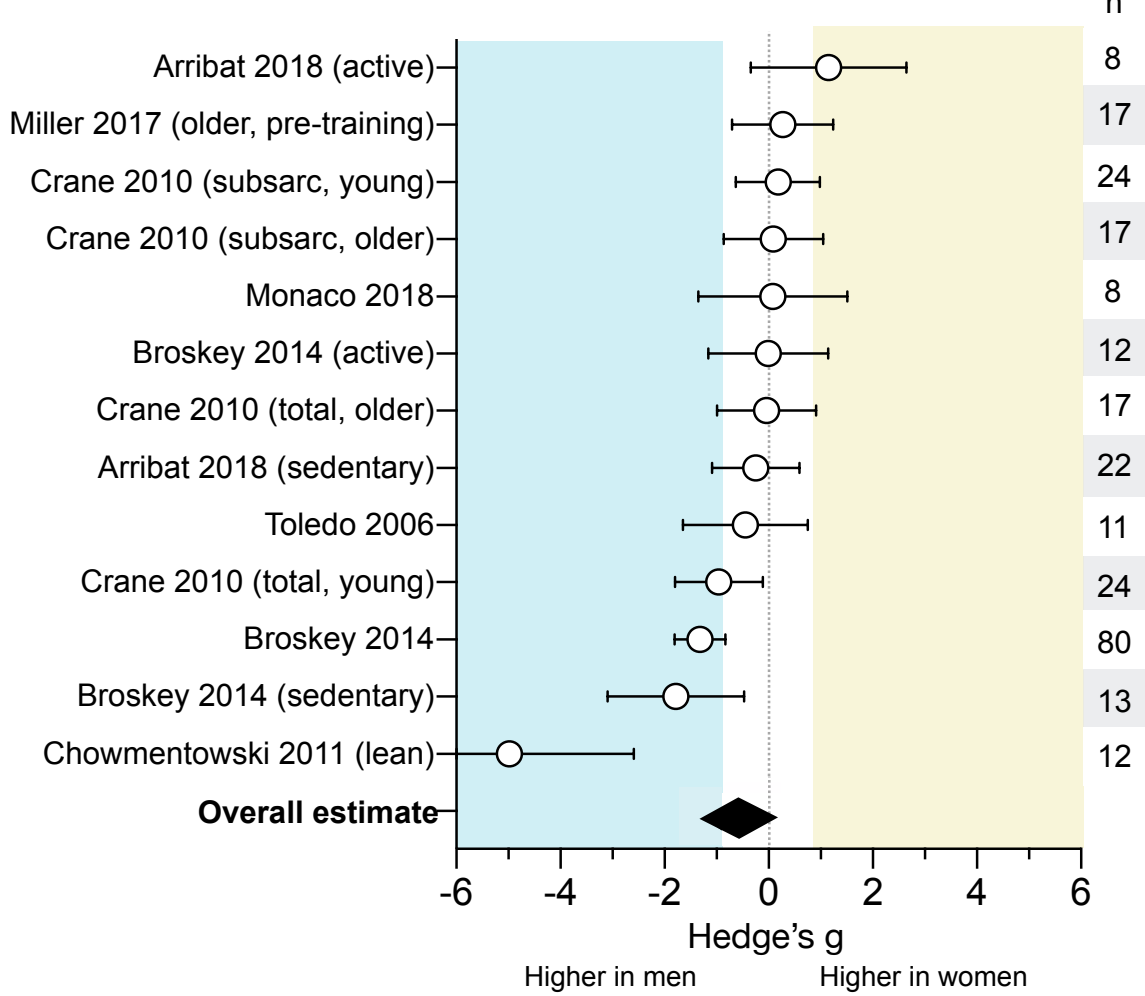

$x^{2}, p=0.13$

b

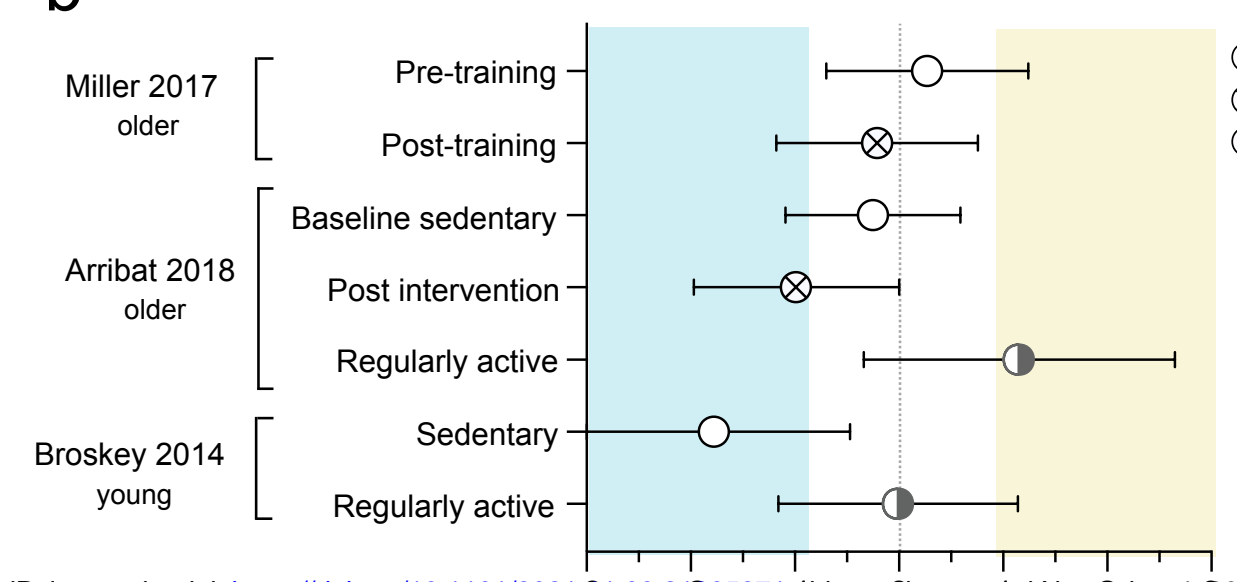

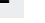

C Miller 2017 (older, pre-training)

Monaco 2018

Crane 2010 (total, old) -

Toledo 2006

Crane 2010 (subsar, young) -

Crane 2010 (subsar, old) -

Crane 2010 (young)

Chowmentowski 2011 (lean)

Overall estimate

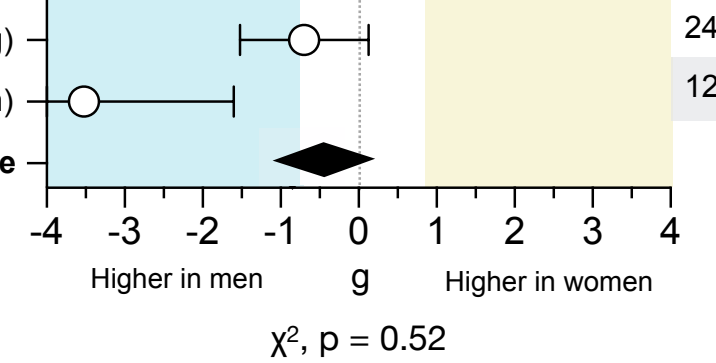

d

Baseline

$\otimes$ Post-training

Active individuals

Miller 2017

older

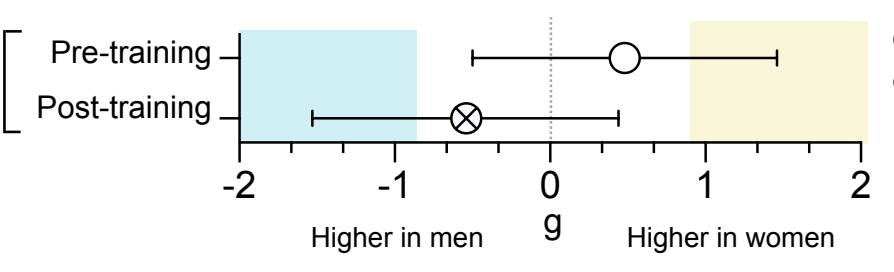

Baseline $\otimes$ Post-training

medRxiv preprint doi: https://doi.org/10.1101/202L31.03.2126587L; this verGion posted Nove2nber 4,3021. The copyright holder for this preprint

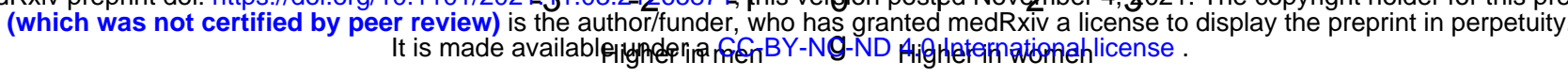

Figure 8 - Skeletal muscle mitochondrial volume density and size lack consistent sex differences.

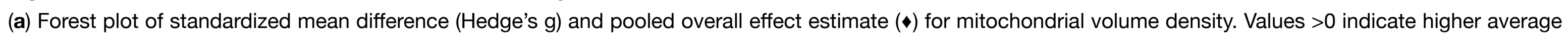

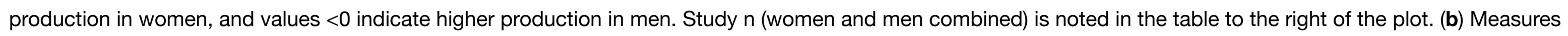

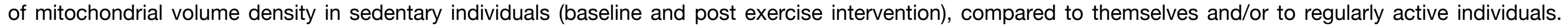

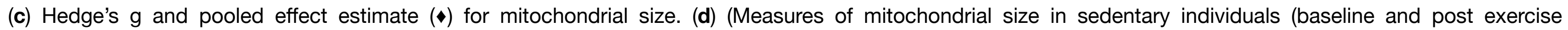
intervention) 

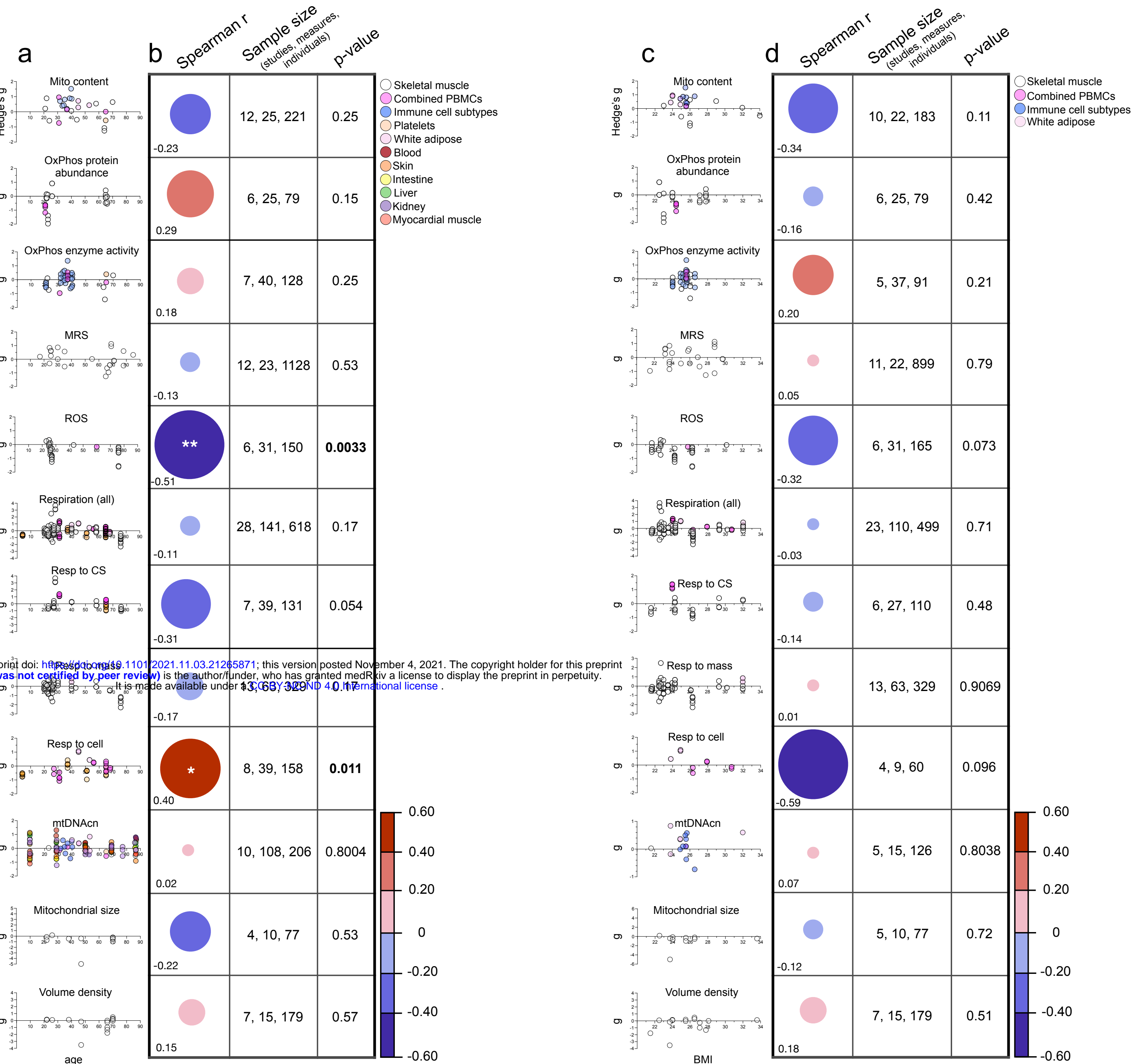

$-0.60$

Figure 9 - Binary sex differences in mitochondrial biology show weak to no association with age and BMI.

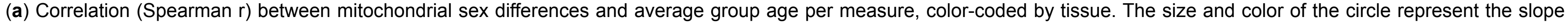

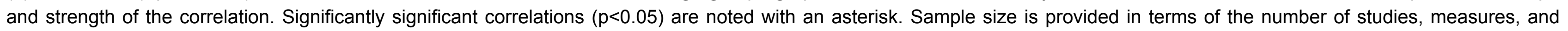
individuals. (b) Spearman $r$ between mitochondrial sex differences and average BMI per measure, with points color-coded by tissue. 
Figure 10
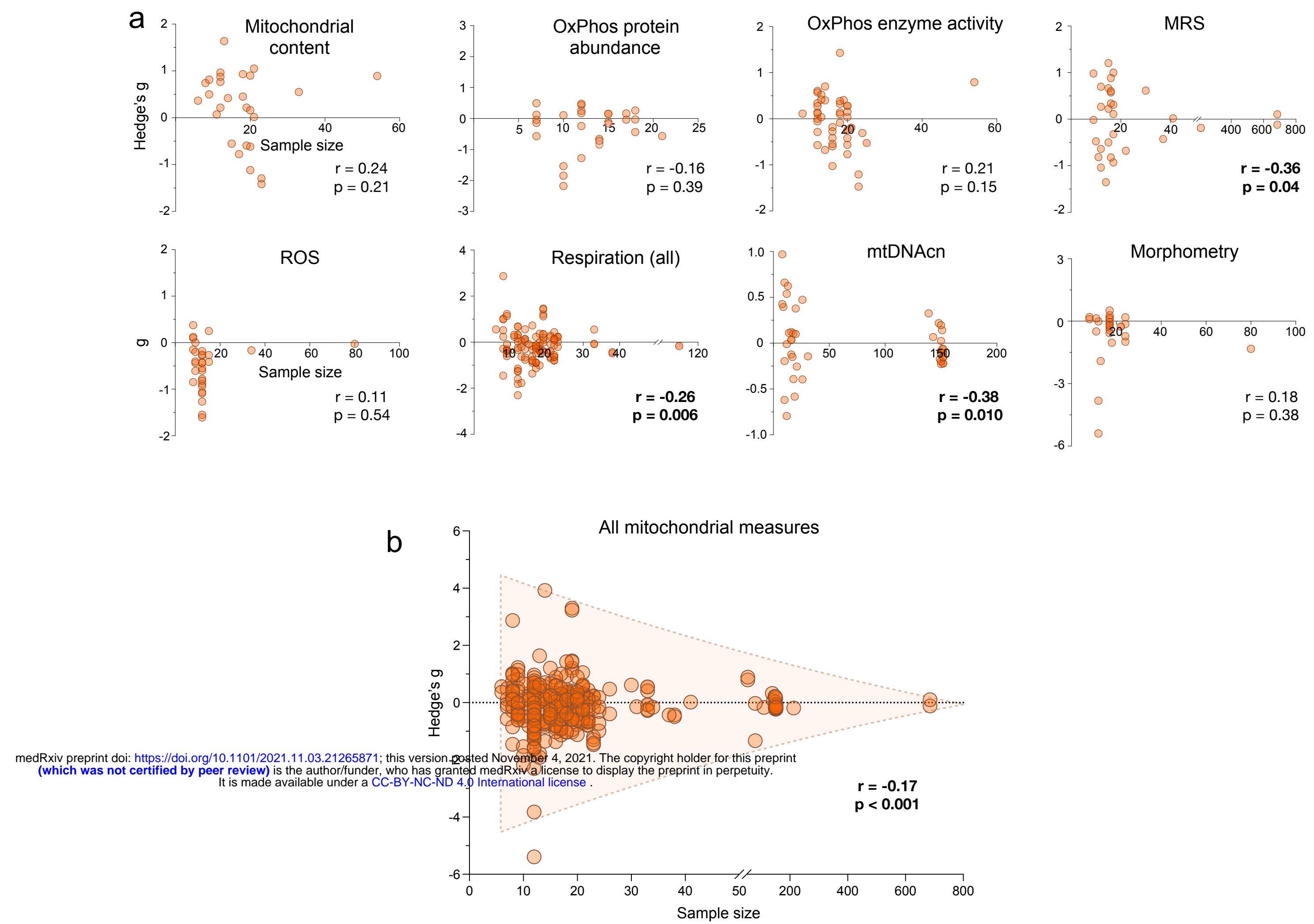

Figure 10 - Observed sex differences in mitochondrial biology are negatively correlated with sample size.

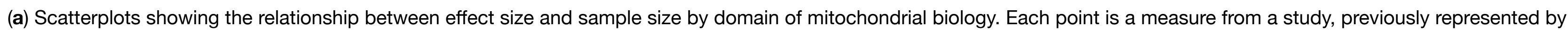

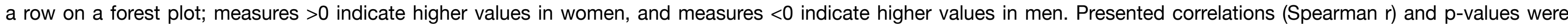

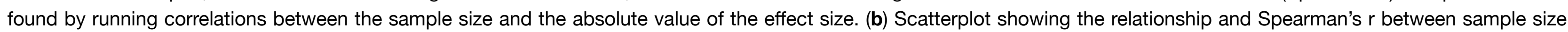
and effect size for all included measures. 